\title{
réalisation d'un remblai fondé sur des vases et argiles molles dans le lac de Tunis
}

par

\author{
J.-L. Bordes \\ H. Guellec \\ Coyne et Bellier
}

\section{Introduction}

Le canal Lac Nord est un des ouvrages les plus importants parmi ceux qui ont été projetés et exécutés, dans le cadre de la tranche prioritaire des travaux d'assainissement du grand Tunis, qui se poursuivent depuis 1972 .

Ce canal est réalisé en bordure du lac de Tunis, à la limite de la basse ville (figure 1). II en reçoit les eaux pluviales et celles de quelques oueds et canaux de drainage avoisinants. II transfère ces eaux vers une station de relèvement, qui permet de les envoyer dans le port, afin de pouvoir les évacuer gravitairement vers la mer par le canal de navigation. II permet d'évacuer par temps sec, les eaux du lac à débit constant vers la mer pour en assurer le renouvellement.

On espère ainsi, en premier lieu, éviter la pollution du lac Nord en empêchant que les eaux pluviales du réseau unitaire ne s'y déversent trop fréquemment (pas plus d'une fois ou deux par an). En second lieu, on cherche à réactiver les eaux du lac par une circulation privilégiée, alimentée depuis la mer par le canal de Kheredine. Enfin, on entend éviter en temps sec, les intrusions d'eau salée dans le réseau d'assainissement.

Le canal est de section trapézoïdale. Sa largeur au plafond est de $28 \mathrm{~m}$. La cote de son radier est à $-0,90$ NGT, la cote des berges à $+1,20$ NGT. Les talus ont des pentes à 2/1. Le niveau d'eau dans le canal en régime normal est à $-0,40 \mathrm{NGT}$, alors que celui du lac est à $+0,10$ NGT en moyenne.

La longueur du canal est d'environ $5000 \mathrm{~m}$. Tandis que sur une longueur d'environ $3500 \mathrm{~m}$, le canal est excavé dans des remblais récents constituant les berges du lac, des contraintes diverses ont exigé que le canal soit implanté sur $1300 \mathrm{~m}$ environ en bordure immédiate du lac. II a fallu réaliser un remblai de $1300 \mathrm{~m}$ de long et de $70 \mathrm{~m}$ de largeur environ, fondé sur plusieurs dizaines de mètres de vase et d'argile molle. On se propose dans le présent article d'exposer comment a été conçu et réalisé ce remblai, en fonction des données particulières du site.

On examinera successivement les données géologiques et géotechniques du site, la conception du remblai et de sa fondation, les conditions de son exécution, son comportement, avant d'exposer les enseignements que l'on peut tirer de ces travaux.

\section{Données générales sur le site}

\subsection{Géologie sommaire du lac de Tunis (figure 2) [1]}

Le lac de Tunis a été le siège d'une sédimentation marine importante à l'époque crétacé oligocène. Des phases de plissements considérables accompagnées par la formation de zones faillées importantes ont provoqué l'exhaussement des dépôts et leur érosion. Une succession de transgressions et de régressions depuis le début du Pliocène a vu le lac de Tunis alors largement ouvert sur la mer, être le siège d'une sédimentation estuarienne due à la Medjerdah qui passait alors entre les collines de la Kasbah et du Belvédère. II y a 50000 ans un grand glissement coupait le cours de la Medjerdah dans la zone d'Oued Ellil et provoquait son détournement vers le Nord dans la plaine deltaïque actuelle. Le futur lac de Tunis toujours ouvert sur la mer était le siège d'une sédimen tation lagunaire, alimentée par les courants venant du Nord et aidée par la subsidence provoquée par des plissements très récents.

Ce n'est que fort tard, bien après le début de l'ère chrétienne que le lac s'est définitivement formé, alors que la mer d'Utique s'était remblayée par suite des apports de la Medjerdah. Rappelons à titre historique que Carthage qui n'était qu'une île avant l'Acheuléen, a été réunie à la côte à la suite d'une régression par un isthme limité. La côte Nord était à $11 \mathrm{~km}$ à l'inté rieur des terres actuelles allant d'Utique à Gamart par le Djebel Nahali. Cette topographie subsistait à l'épo 


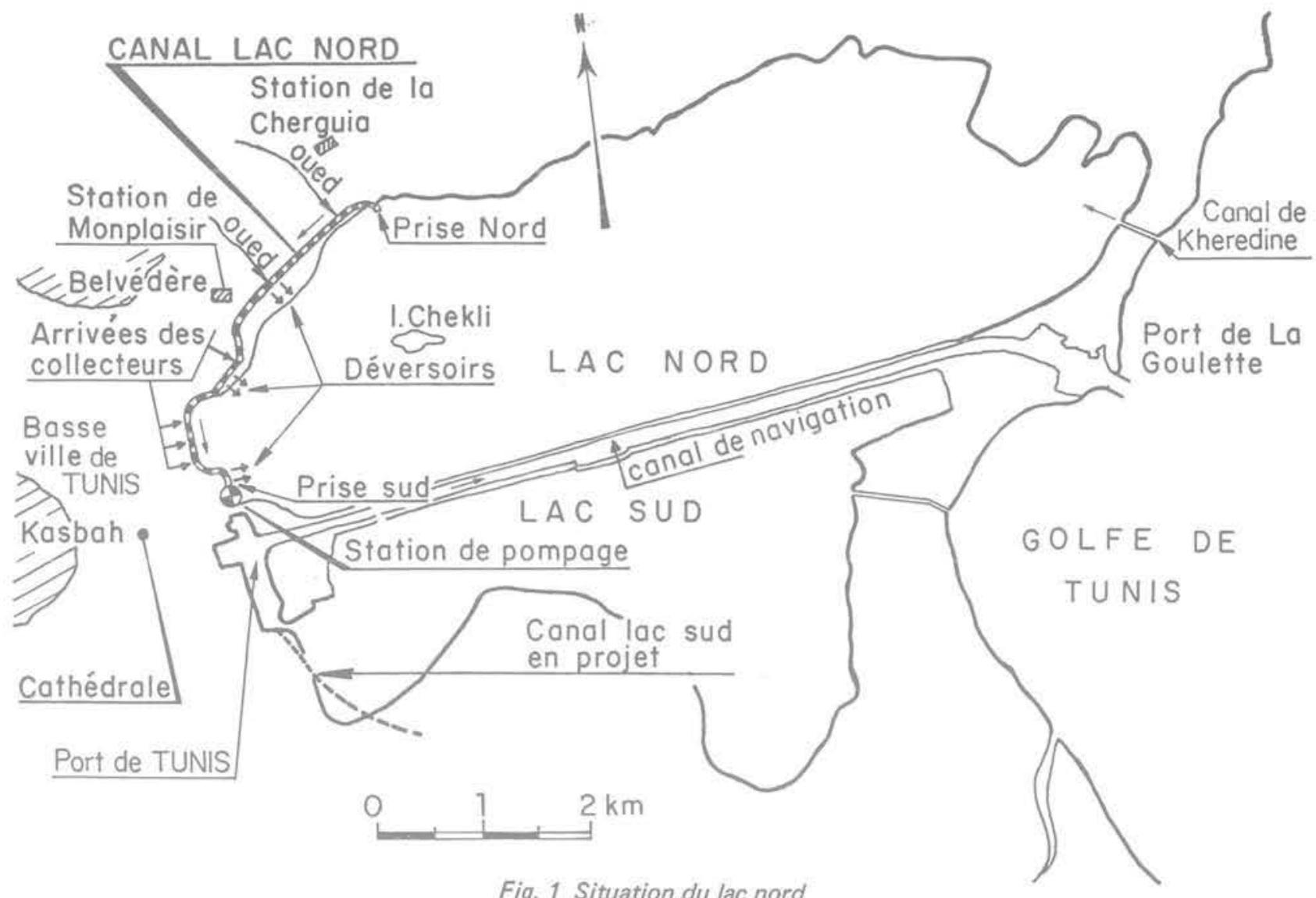

Fig. 1 Situation du lac nord

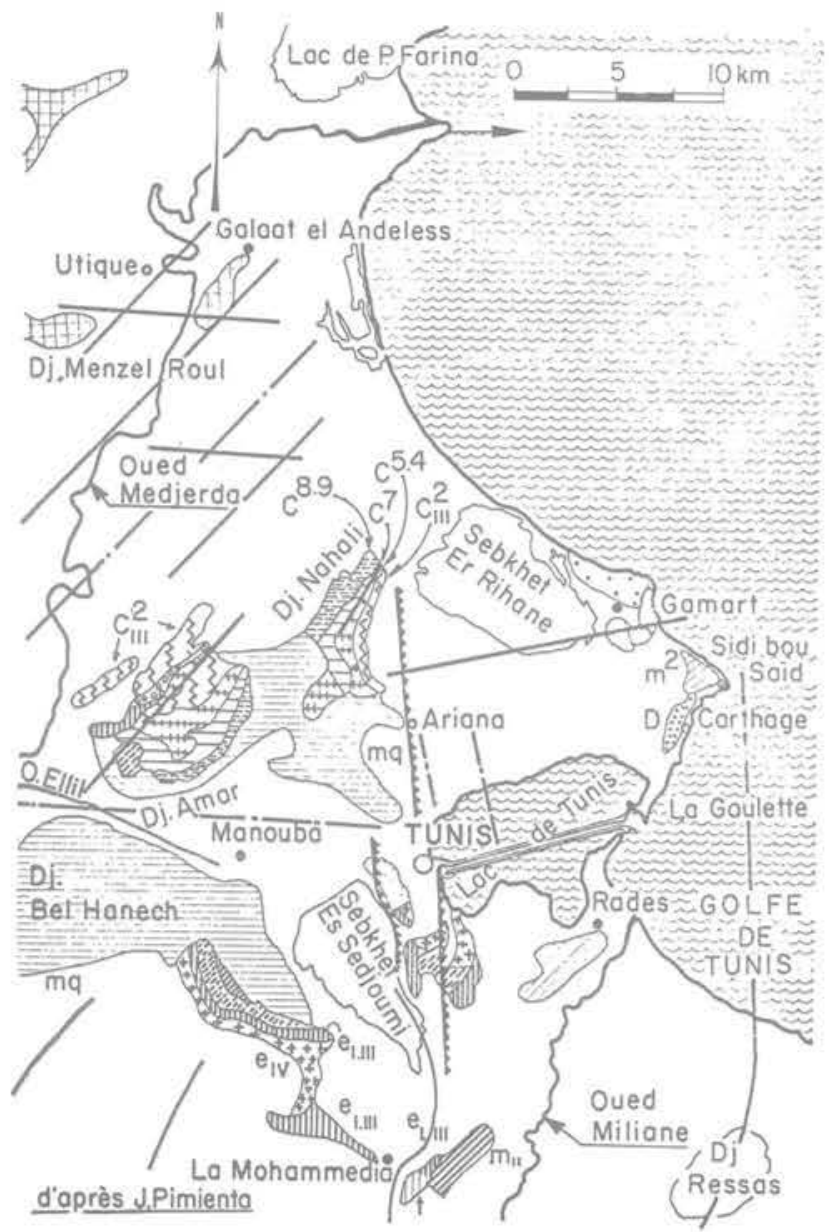

Fig. 2 Carte géologique d $\because \because$ Dune octuelle

D

$\mathrm{P}^{\mathrm{bc}}$ 曲曲 Pliocène marin supérieur

$\mathrm{mq}$ Plioquaternaire continental

$\mathrm{m}^{2}$ Miocène, marnes bigorrées

$\mathrm{m}_{u}$ TIIIII Oligocène

e'

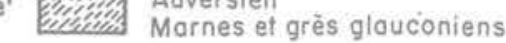

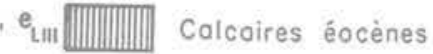

iv

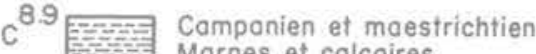

$c^{7} \rightleftharpoons \begin{aligned} & \text { Sénonien inférieur } \\ & \text { Mornes }\end{aligned}$

$c^{54} \ldots \begin{aligned} & \text { Cenomonien } \\ & \text { Marno_calcoire }\end{aligned}$

$2{ }^{2}$ ह Crétace inférieur

Marnes, schistes et calcaire

J $\because \because \frac{\text { Jurassique }}{}$

t $\square$ Keuper

Axes anticlinaux
- Axes synclinaux
- Failles récentes et actuelles


que romaine et explique les positions stratégiques relatives d'Utique et de Carthage.

La fermeture définitive du lac vraisemblablement après le $16^{\mathrm{e}}$ siècle lui a donné son faciès lacustre.

II est important de noter qu'à l'époque du Villafranchien au tout début du quaternaire, une croûte calcaire s'est formée sur une grande surface en tête des dépôts. La déformation de cette croûte calcaire traduit l'existence de plissements ultérieurs résultant d'une activité tectonique qui n'a jamais cessé jusqu'à aujourd'hui.

La vieille ville de Tunis se trouve sur une butte constituée par les terrains gréseux et argileux du Pliocène continental et du Miopliocène (Pontien). La structure synclinale dont fait partie la colline de la Kasbah et dont l'axe est orienté Sud-Ouest, Nord-Est, se "noie" " au pied du Belvédère pour ne réapparaître qu'à Carthage. Cependant, il subsiste une ride reliant ces deux émergences et constituant la séparation entre le lac de Tunis et le Sebkhet Ariana. La croûte calcaire légèrement plissée, reflète le tectonisme quaternaire. Un gonflement anticlinal parallèle à la barrière de Tunis la fait affleurer sur l'île Checkli.

Cette croûte, suite à la transgression marine qui a donné lieu à la naissance de la lagune, se trouve en grande partie sous les vases.

En fonction de l'histoire géologique du lac et en ne considérant que les couches supérieures, c'est-à-dire les quelques dizaines de mètres depuis la surface, on ne s'étonnera pas de ne rencontrer que des sols fins, des vases, argiles ou sables, classés en fonction des régimes et de la morphologie de la Medjerdah pour la période de l'alluvionnement fluviatile ou estuarien, avant que ne lui succèdent les périodes de dépôts lagunaires puis lacustres.

Mais les transgressions et régressions successives que l'on peut remarquer à l'oxydation de différents dépôts ont provoqué des modifications dans la granulométrie des dépôts, ainsi que dans leur résistance par consolidation par dessiccation en surface au moment de l'apport. Dans l'ordre des altérations lors des phases d'émersion, il ne faut pas oublier dans la partie Nord du lac le cas important de la croûte calcaire déjà mentionnée résultant de la cimentation des dépôts existants.

\subsection{Situation géologique du canal et strati- graphie}

Le tracé du canal "Lac Nord " décrit un arc de cercle. II commence au droit de la station La Cherguia pour aboutir en longeant la zone de l'Esplanade dans le canal de navigation allant du port de Tunis à celui de La Goulette.

Le tracé du canal recoupe la zone de l'ancien estuaire de la Medjerdah et on peut a priori distinguer deux zones :

- celle correspondant à la partie du canal qui longe la rive de l'ancien isthme de Carthage dont l'axe appartient à la structure anticlinale Kasbah-Carthage: - celle orientée Nord-Sud correspondant à la partie du canal perpendiculaire à ce dernier axe géologique et qui se trouve à $1000 \mathrm{~m}$ environ de l'endroit du rivage au début de l'ère chrétienne, qui passait alors au droit de la cathédrale.

La stratigraphie de la zone a été établie par de nombreux sondages exécutés ([3] à [6]) pour les

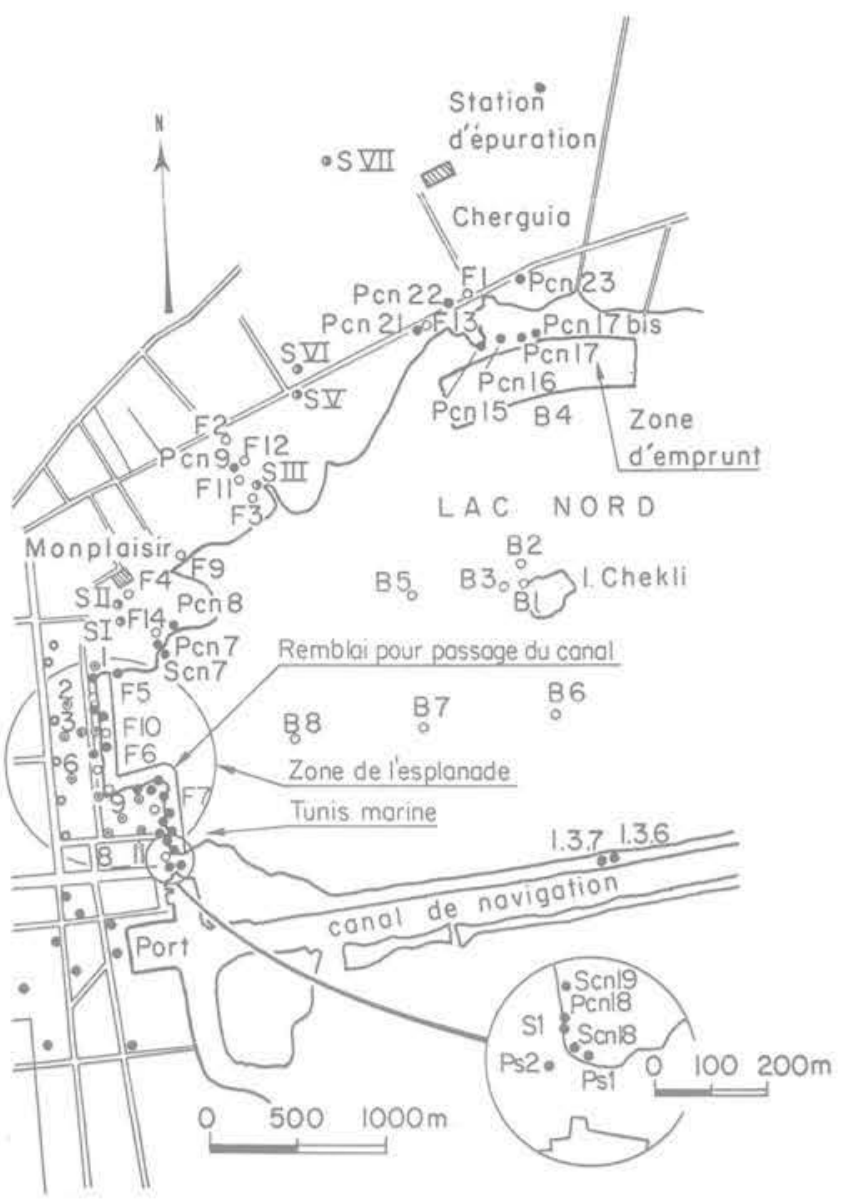

Fig. 3 Sondages et essais in situ dans la zone du lac; implantation du remblai

besoins de l'étude du canal ou d'ouvrages existant sur les bords du lac. Pour le canal, on a exécuté en deux campagnes, dans la zone la plus délicate, celle de l'Esplanade, environ 50 essais de pénétration statique jusqu'à 30 mètres de profondeur et autant d'essais au scissomètre jusqu'à 20 mètres de profondeur. On doit noter que les renseignements dont on pouvait disposer par ailleurs sont nombreux jusqu'à 15 à $20 \mathrm{~m}$ de profondeur. La figure 3 donne un aperçu des reconnaissances faites dans la zone du lac. Elle ne saurait être considérée en aucun cas comme le fruit d'une recherche exhaustive. Elle rappelle l'implantation de sondages et essais in situ, faits depuis 1966 pour la plupart d'entre eux, pour l'étude du canal lac Nord ou de divers aménagements connexes relatifs à l'assainissement du lac.

Dans la première partie du canal suivant son axe, depuis l'amont jusqu'à son entrée dans la zone de I'Esplanade, les coupes stratigraphiques des sondages montrent d'une façon très concordante, sous 1 à $2 \mathrm{~m}$ de remblais, une alternance de couches de sables, d'argiles et de vases. On peut mettre en évidence plusieurs phases d'émersion grâce à l'oxydation des niveaux de sable (sable jaune) et d'argile (argile bariolée), entrecoupés par des sables gris et des vases et argiles noires et grises. Les couches de sable, en particulier la couche supérieure, peuvent atteindre des épaisseurs importantes. Dans la zone d'emprunt, on trouve une épaisseur de sable de 10 à $12 \mathrm{~m}$ comprenant 3 à $4 \mathrm{~m}$ de sable jaune très pur et 6 à $7 \mathrm{~m}$ de sable gris avec quelques passées argileuses. Le toit des sables aux alentours du sondage F13 et dans la zone d'emprunt se trouve très près de la surface. Celui-ci plonge ensuite dans la direction 


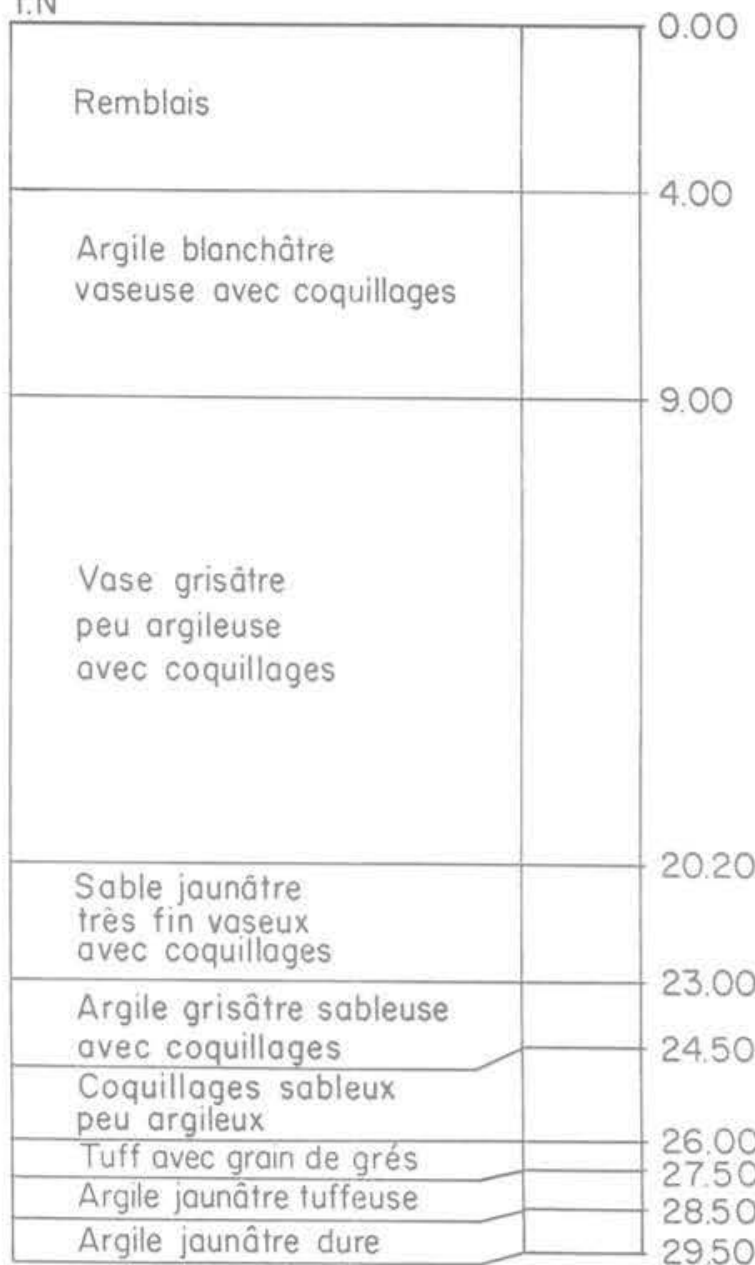

\section{4a Coupe stratigraphique}

\section{Fig. 4 Sondage $51 / 74$}

Est pour se situer à $10 \mathrm{~m}$ environ de profondeur au droit du sondage F14.

Dans la zone de l'Esplanade, c'est-à-dire dans la deuxième partie du canal, on ne rencontre plus, près de la surface, de couche de sable d'épaisseur notable. On traverse une épaisse couche de vase et d'argile molle jusqu'à environ le niveau - 20.00 NGT. Au-delà de ce niveau, on rencontre des argiles sableuses plus consistantes avec quelques niveaux sableux de faibles épaisseurs. Les argiles sont plus ou moins tuffeuses et présentent des traces d'oxydation ( $-23 \mathrm{NGT}$ )

La croûte calcaire se présente en de nombreux points avec une épaisseur de 30 à $70 \mathrm{~cm}$ d'épaisseur. Cette croûte est extrêmement dure. Son extension est importante et elle doit être considérée comme continue en particulier sur les bords du lac dans la rive Nord sous les remblais ( 1 à $2 \mathrm{~m}$ de profondeur) ou sous les vases du lac dans la zone d'emprunt (0,50 $\mathrm{m}$ de profondeur). On trouve cette même croûte à $14 \mathrm{~m}$ de profondeur sous les vases dans les sondages B8, B7 et B6. Des auteurs [1] la signalent à une profondeur de l'ordre de $11 \mathrm{~m}$ sous les berges du canal de navigation. Enfin, des niveaux de tuf, rognons de grès, etc. sont indiqués par les sondeurs dans les sondages F7, F8, $S 1 / 74$ à $22 \mathrm{~m}$ de profondeur et à quelques 20 à $25 \mathrm{~m}$ de profondeur pour une partie des sondages exécutés pour l'étude de l'aménagement du quartier de la petite Sicile.

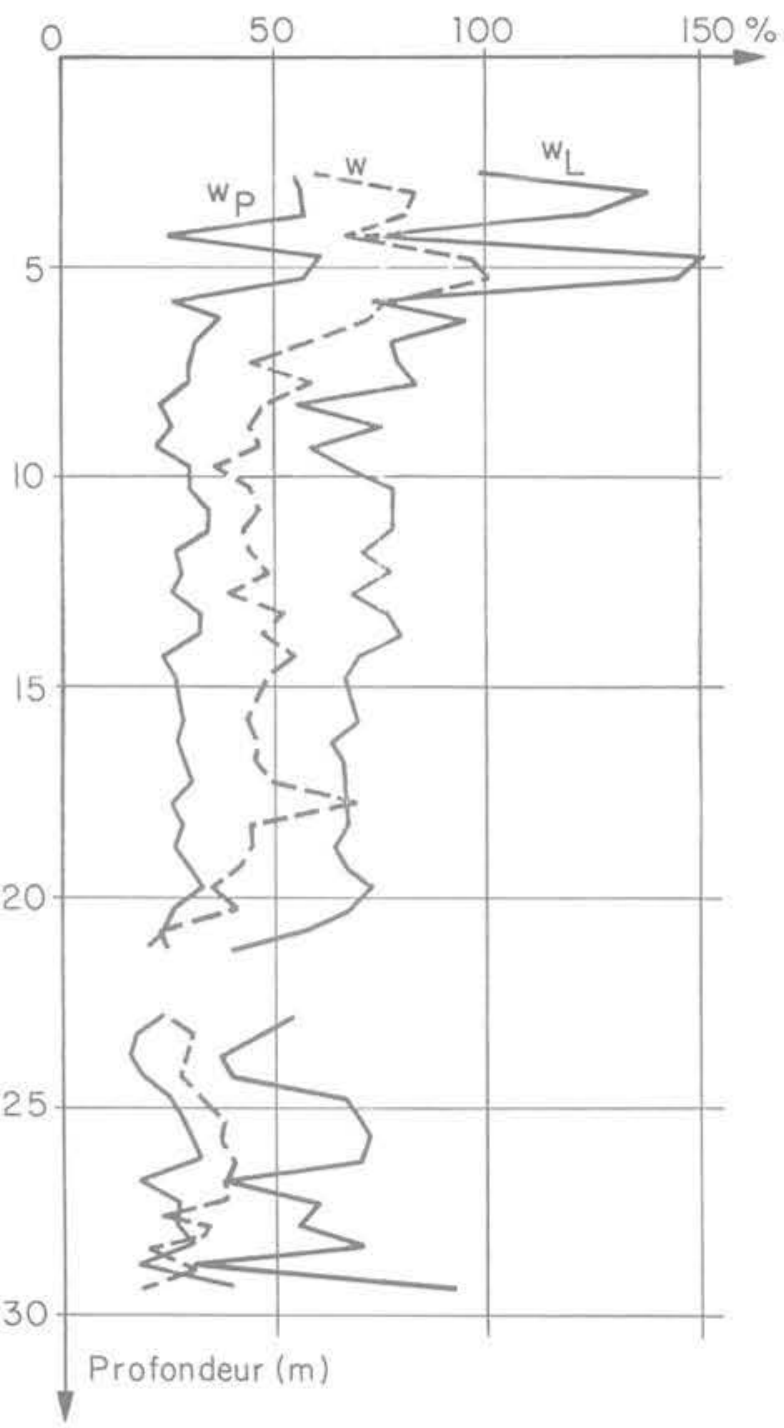

4b. Limites d'A tterberg et teneur en eau naturelle

Aussi les coupes stratigraphiques, sur le tracé du canal suivant l'axe F5, F6, F7, F8, qui montrent une épaisseur de vase et d'argile molle de l'ordre de 15 à $20 \mathrm{~m}$ au-dessus des premiers niveaux oxydés, traduisent-elles l'existence dans la zone de l'Esplanade et du port d'un surcreusement, soit tout simplement d'un plongement dû au mouvement tectonique du toit de la première couche de sables oxydés. La pente générale depuis l'extrémité amont du canal varie de 5 à $10 \%$. Des vérifications de ce niveau seraient nécessaires, mais l'établissement d'une stratigraphie aussi détaillée présentait un intérêt limité pour l'étude du canal. Par contre, on peut affiner la stratigraphie des vases et argiles entre le fond du lac et le niveau -20 NGT. Ce point qui relève plus de l'aspect géotechnique du problème que de son aspect géologique, sera abordé dans l'étude des fondations du remblai dans le paragraphe suivant.

\subsection{Données géotechniques sur les sols}

\subsubsection{Généralités}

En complément aux essais in situ mentionnés dans le paragraphe précédent, l'étude du canal a demandè l'exécution d'un certain nombre d'essais qui sont venus se joindre à une documentation déjà abondante. Le site de Tunis difficile au plan géotechnique a 


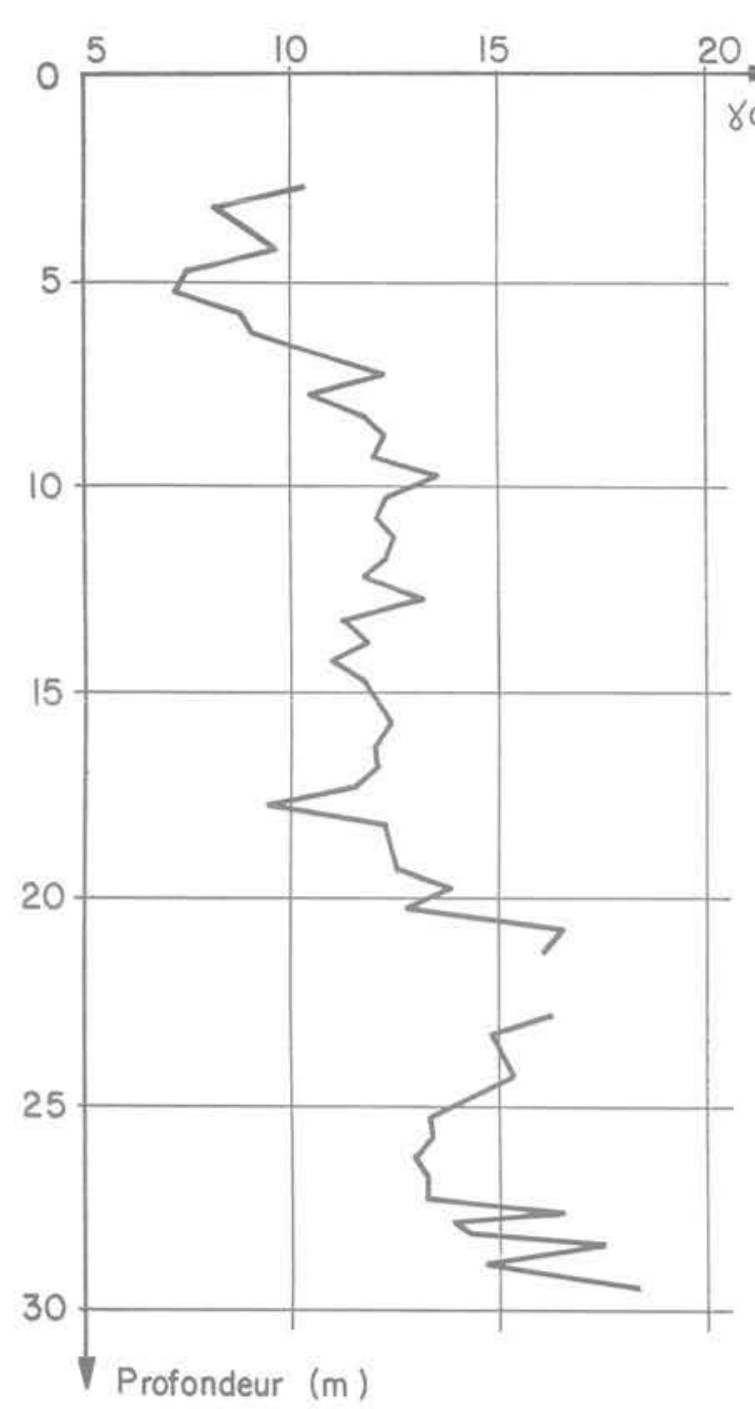

4c Poids volumique sec

4e Essai pénétrométrique

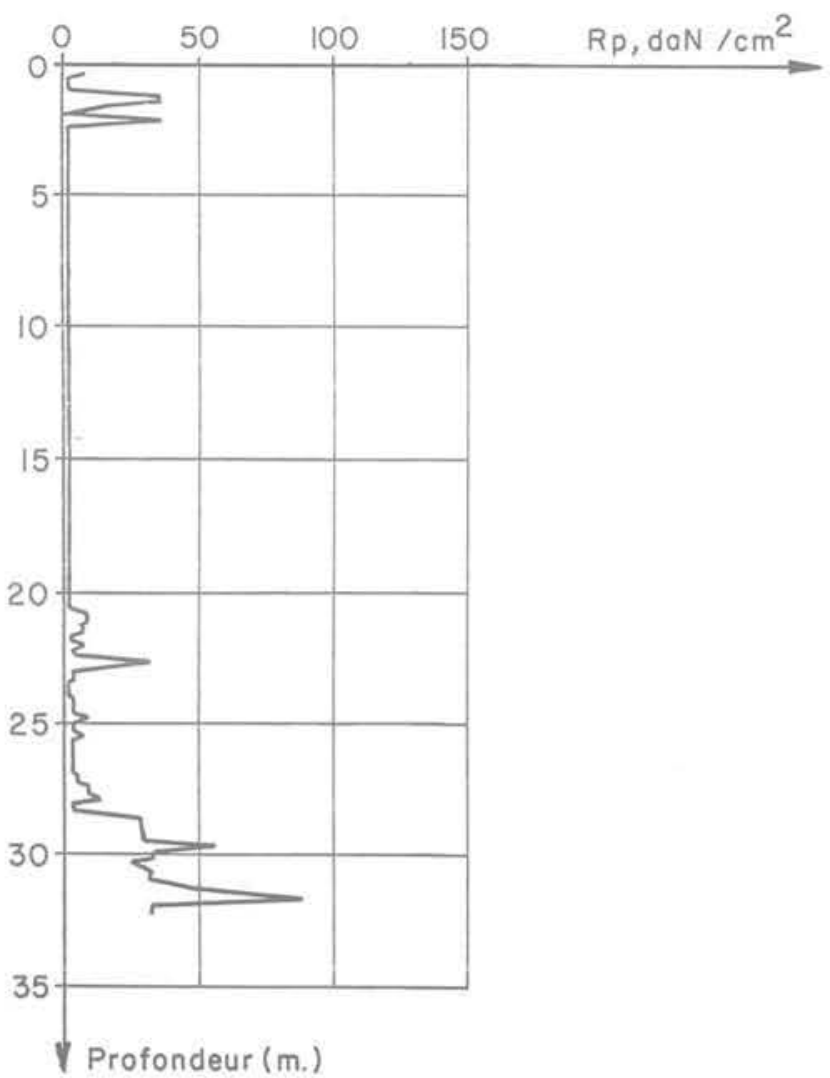

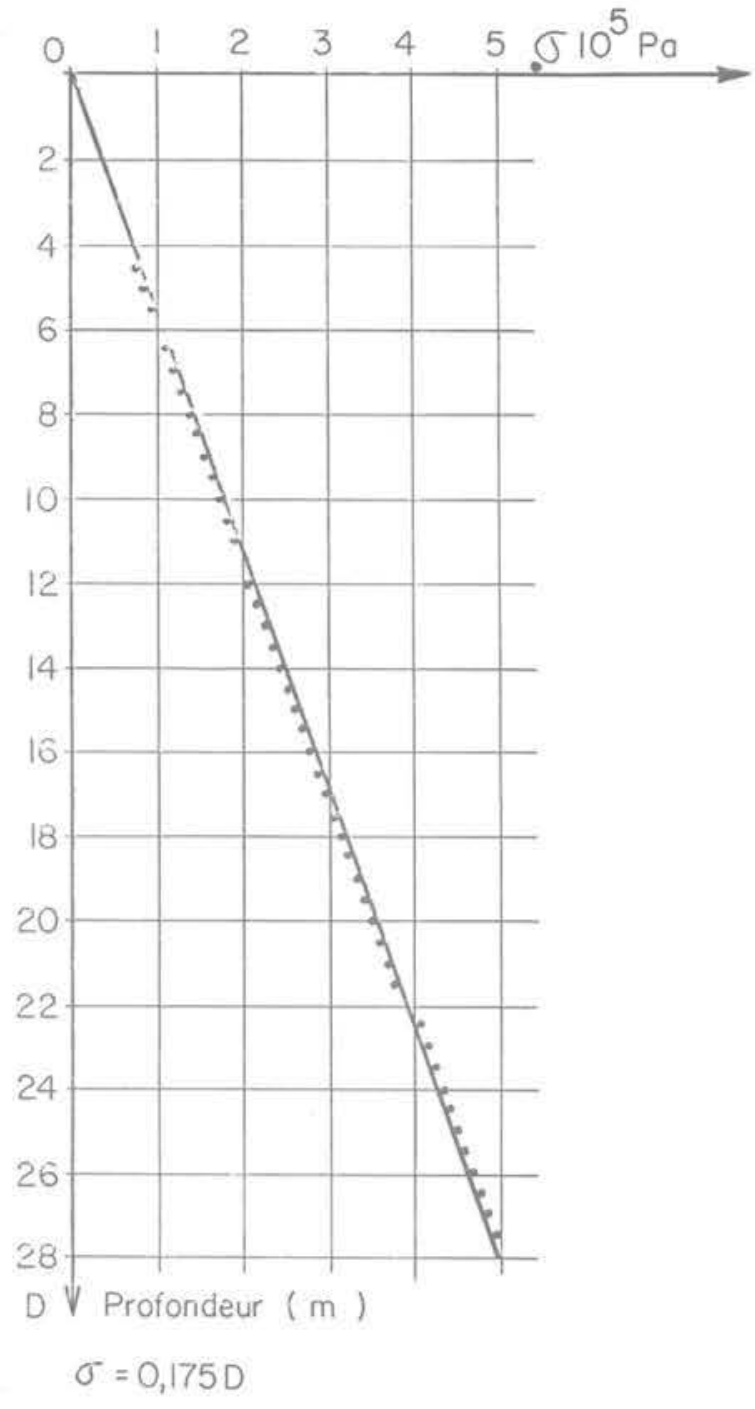

4d Contrainte verticale totale

$4 f$ Essais scissométriques

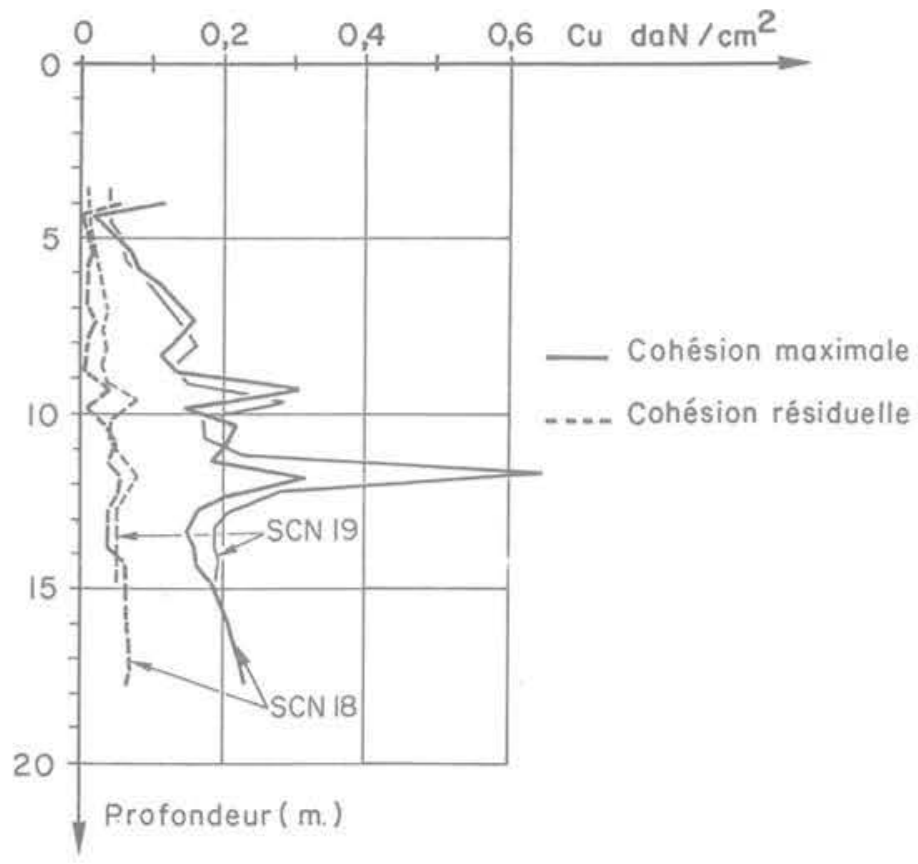


fait l'objet d'études de la part de géotechniciens éminents. On se propose de faire une synthèse modeste, réduite aux matériaux rencontrés dans la zone de l'Esplanade.

\subsubsection{Vases et argiles}

Identification

Pour les sondages exécutés antérieurement à 1975 . on a les corrélations suivantes entre $I_{p}$ et $w_{L}$.

Tableau 1

\begin{tabular}{|c|c|c|c|c|c|}
\hline $\begin{array}{l}\text { Sondages } \\
\text { et dates }\end{array}$ & $\begin{array}{c}\text { Nombre } \\
\text { de mesures }\end{array}$ & $\begin{array}{l}\text { Profondeur } \\
\text { en mètres }\end{array}$ & $\begin{array}{l}\text { Relation } \\
\mathrm{I}_{D} \mathrm{~W}_{\mathrm{L}}\end{array}$ & $\begin{array}{l}\text { Coefficient de } \\
\text { corrélation }\end{array}$ & $\begin{array}{l}\text { Valeurs } \\
\text { extrêmes }\end{array}$ \\
\hline $\begin{array}{l}\mathrm{S} 1 \text { à } \mathrm{S} 15(1967) \\
4.1 \text { à } 4.6(1968) \\
1.3 .8 / 1.3 .9 / 3.1\end{array}$ & $\begin{array}{l}65 \\
47\end{array}$ & $\begin{array}{l}0 \text { à } 8 \mathrm{~m} \\
0 \text { à } 10 \mathrm{~m}\end{array}$ & $\begin{array}{l}I_{p}=0,74\left(w_{L}=11\right) \\
I_{P}=0,63\left(w_{L}=5\right)\end{array}$ & $\begin{array}{l}0,93 \\
0,91\end{array}$ & $\begin{array}{l}23 \text { à } 150 \\
35 \text { à } 135\end{array}$ \\
\hline $\left.\begin{array}{c}3.2 / 1.101 / 1.111 \\
(1968)\end{array}\right\}$ & 37 & 0 à $10 \mathrm{~m}$ & $I_{P}=0,73\left(w_{L}-13\right)$ & 0,96 & 27 à 152 \\
\hline $\left.\begin{array}{c}\text { mêmes sondages } \\
\text { que ci-dessus } \\
4.1 \text { et } 4.6(1968)\end{array}\right\}$ & $\begin{array}{l}37 \\
+ \\
21\end{array}$ & $\begin{array}{l}0 \text { à } 10 \mathrm{~m} \\
10 \text { à } 20 \mathrm{~m}\end{array}$ & $I_{P}=0,75\left(w_{L}-12\right)$ & 0,97 & 52 à 140 \\
\hline
\end{tabular}

Sur le tracé du canal dans la zone la plus médiocre, le sondage S1 exécuté près de Tunis Marine en 1974 $(S 1 / 74)$, figure 4 , permet d'avoir un ensemble de résultats de mesures sur des échantillons non remaniés. Bien qu'il faille, semble-t-il, comme on le verra par la suite, distinguer les couches situées entre 0 et $10 \mathrm{~m}$ de profondeur de celles entre 10 et $20 \mathrm{~m}$, puis de celles au-delà, une corrélation $I_{p} w_{L}$ a donné pour 40 mesures entre 0 et 20 mètres de profondeur:

$I_{p}=0.68\left(w_{L}-9\right) \quad 23 \leqslant w_{L} \leqslant 93$ voir figure 5

Dans cette corrélation, on a éliminé les matériaux organiques des premiers mètres, qui, comme le montre leur position dans l'abaque de plasticité de Casagrande, sont de nature différente des matériaux sousjacents.
Le graphique $4 b$ de la figure 4 , donne les limites de liquidité et de plasticité mesurées dans le sondage $S 1 / 74$. On y trouve également les teneurs en eau naturelle. Mais ces teneurs en eau n'ont pas été mesurées directement mais seulement estimées à partir du poids volumique sec des échantillons.

En 1979, plusieurs sondages carottès ont été exécutés jusqu'à $20 \mathrm{~m}$ de profondeur sur le tracé du canal. Les points représentatifs dans l'abaque de Casagrande des échantillons prélevés à cette dernière occasion sont indiqués sur la figure 5 . On a la relation :

$$
I_{p}=0,72\left(w_{L}-10.5\right) \quad 35 \leqslant w_{L} \leqslant 81 \quad r^{2}=0,97 \text {. }
$$

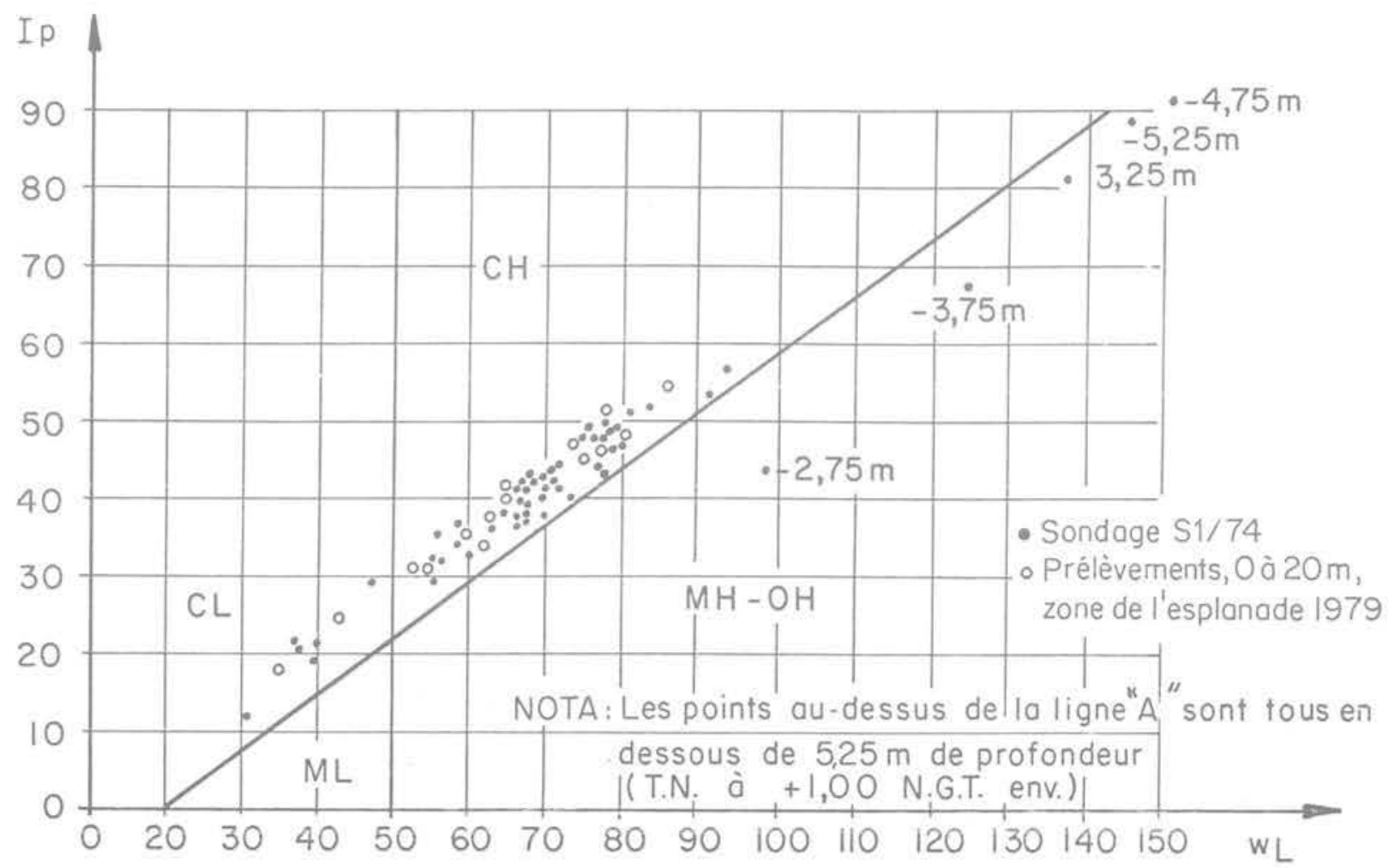

Fig. 5 Points représentatifs des prélèvements dans les vases et argiles molles dans l'abaque de Casagrande 


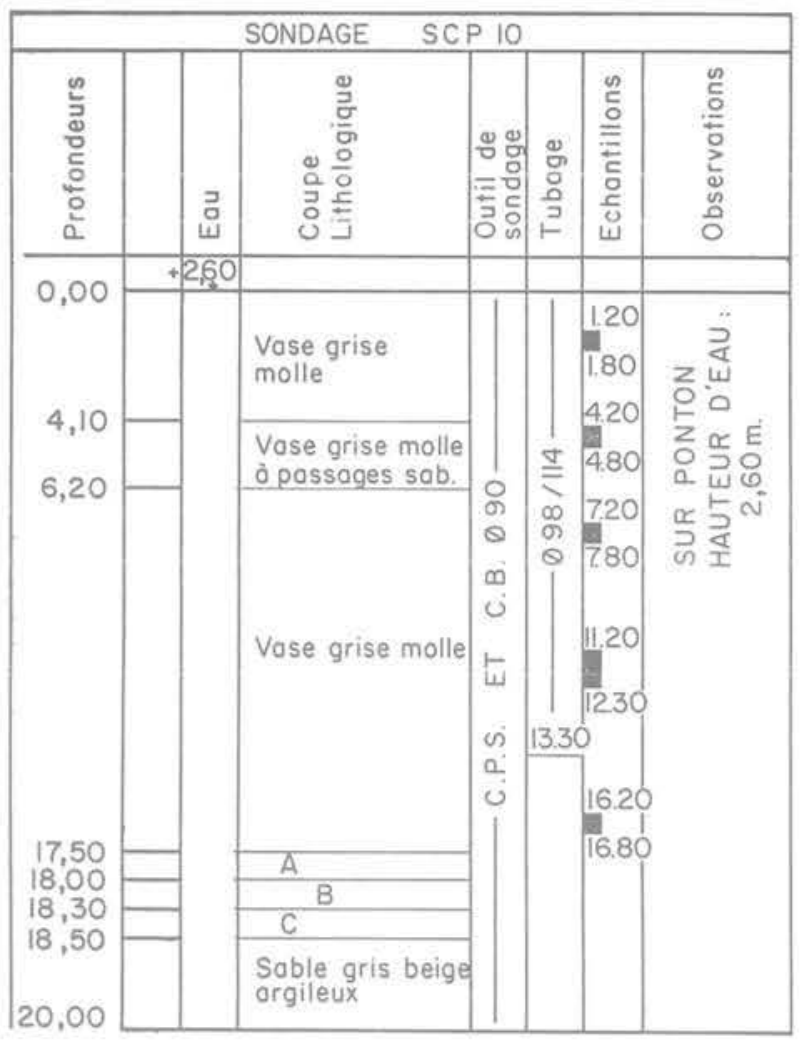

A : Argile gris joune sableuse

B : Sable jaune grossier, coquillier

C + Sable gris. beige argileux

6 a Coupe de sondage
Granulométrie et activité

II va sans dire que les matériaux rencontrés sont constitués de particules extrêmement fines. La quasi totalité de celles-ci ont un diamètre inférieur à 80 microns.

Peu de sédimentométries ont été faites. Aussi l'activité est-elle mal connue. Pour les sondages anciens S1 à $\mathrm{S} 15$ de 1967, jusqu'à $8 \mathrm{~m}$ de profondeur, l'activité était comprise entre 1,06 et 4,6 ; la valeur moyenne étant 2,5 . Pour le nouveau sondage $S 1 / 74$, on note quelques valeurs comprises entre 0,30 et 1,95 . II semblerait que l'activité diminue avec la profondeur. Au-delà de $1.5 \mathrm{~m}$ toutes les valeurs sont inférieures à 1 . On ne dispose pas malheureusement d'analyses minéralogiques faites spécialement sur des échantillons prélevés dans le sondage S1. Toutefois, des études antérieures faites ([1] et [2]) sur les vases permettent d'affirmer qu'il s'agit de dépôts contenant beaucoup de calcaire et que les minéraux argileux sont constitués en grande partie par de l'illite et de la kaolinite.

\section{Poids volumique sec}

Sur les échantillons prélevés dans les années antérieures à 1975, on retiendra qu'entre 0 et $8 \mathrm{~m}$ de profondeur pour 52 échantillons de vases et argiles. on a une valeur moyenne de $9.1 \mathrm{kN} / \mathrm{m}^{3}$, un écart-type de 1,6 . Les valeurs extrêmes sont 6,5 et $11,9 \mathrm{kN} / \mathrm{m}^{3}$.

Les échantillons de matériaux plus sableux au nombre de 11 ont un poids volumique moyen de $14,6 \mathrm{kN} / \mathrm{m}^{3}$, un écart-type de 1,9 , les valeurs extrêmes étant de 13,0 et $18.5 \mathrm{kN} / \mathrm{m}^{3}$.

Fig. 6 Coupe de sondage, essai pénétrométrique et scissométrique

6b Essai pénétrométrique, essai scissométrique - P5

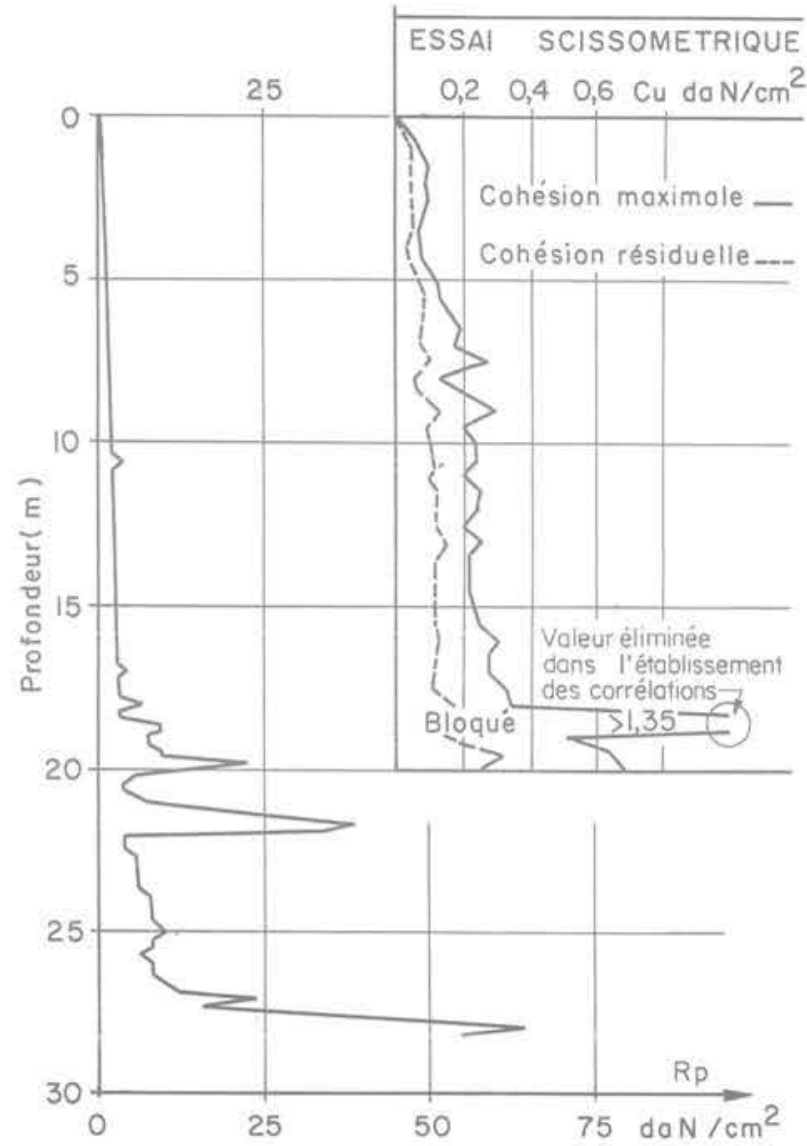

6c Essai pénétrométrique, essai scissométrique - P4

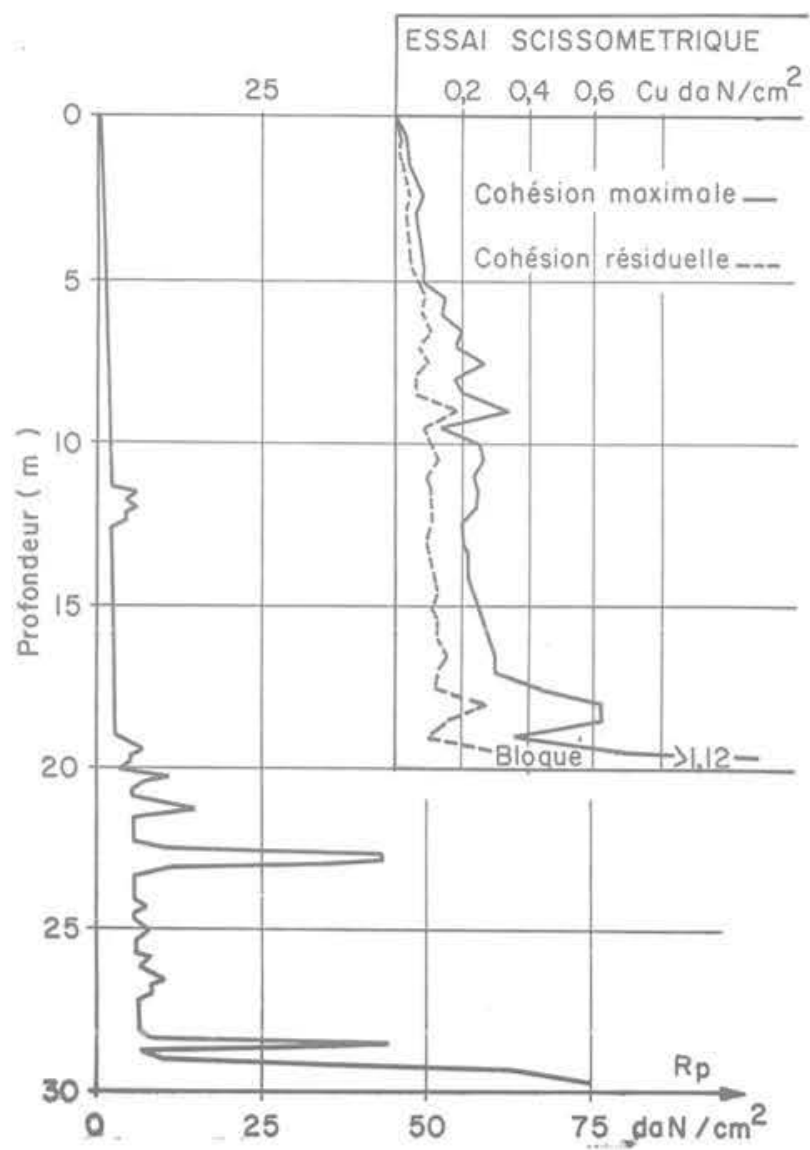


Sur la figure 4 , le graphique $4 c$ donne les valeurs du poids volumique sec en fonction de la profondeur, mesuré sur des échantillons prélevés dans le nouveau sondage S1/74.

Sur la même figure 4 , le graphique 4 d pour le même sondage donne la valeur de la contrainte verticale totale en fonction de la profondeur.

\section{Résistance à la rupture}

Compte tenu de la faiblesse des caractéristiques mécaniques auxquelles on s'attendait, celles-ci ont été mesurées pour les besoins particuliers de l'étude du canal, au moyen d'essais au pénétromètre statique avec mesure du frottement latéral jusqu'à 30 mètres de profondeur et d'essais au scissomètre jusqu'à 20 mètres. Deux campagnes de reconnaissances ont été faites. On a utilisé à cet effet un pénétromètre Gouda de 10 tonnes, un scissomètre Geonor H 250 (1 $1^{\text {re }}$ campagne) et un scissomètre Simec $\left(2^{\circ} \mathrm{cam}\right.$ pagne). La figure 4 , graphiques $4 a, 4 e$ et $4 \mathrm{f}$ et la figure 6 donnent à titre d'exemples les résultats de coupes de sondages, essais pénétrométriques et essais scissométriques dans une même zone (figure 3 ).

Dans les argiles très molles, seuls les essais scissométriques ont permis de mesurer les variations de résistance mécanique. La figure 7 montre les courbes enveloppes des valeurs minimales et maximales mesurées lors de la deuxième campagne. On a éliminé parmi les valeurs maximales, celles correspondant manifestement à des niveaux sableux ou surconsolidés comme indiqués figure 6 .

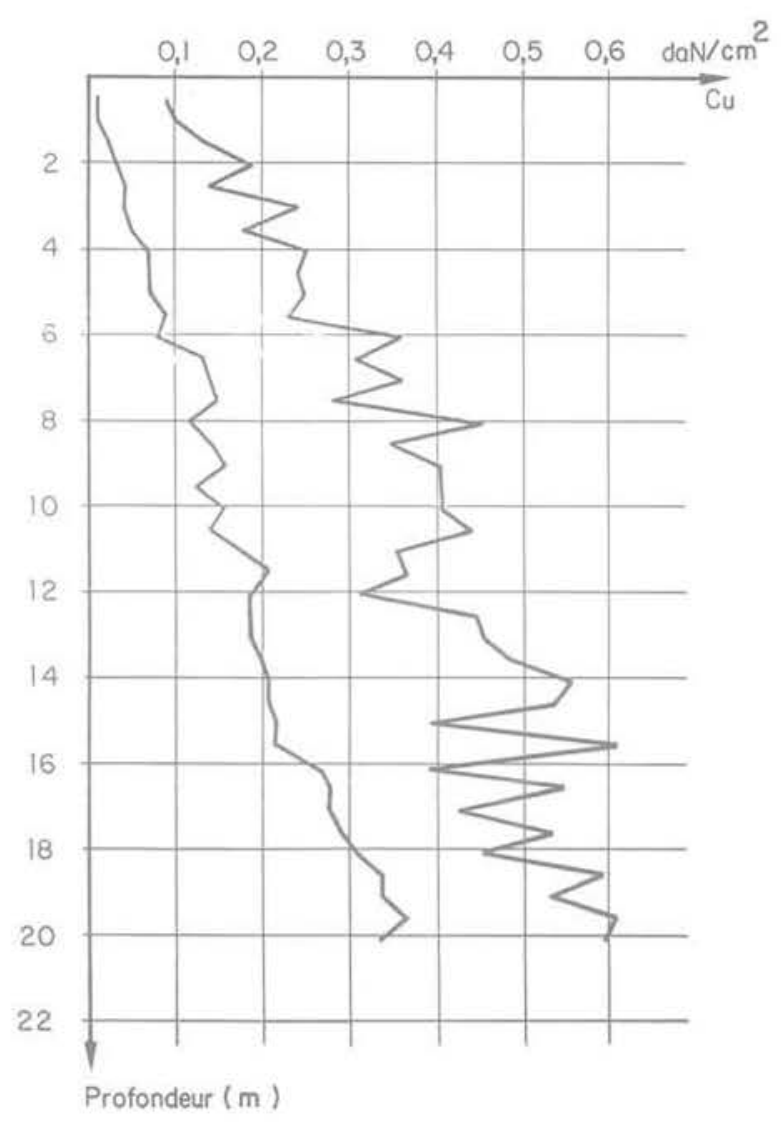

Fig. 7 Essais scissométriques dans les vases et argiles molles, courbes enveloppes des valeurs minimales et maximales
Rappel math 1

Une corrélation Cu-profondeur, donne :

(Cu en daN $/ \mathrm{cm}^{2}, \mathrm{D}$ en mètres) :

a) valeurs maximales $\mathrm{Cu}=0,14+0,023 \mathrm{D}, r^{2}=0,84$;

b) valeurs minimales $\mathrm{Cu}=0,07+0,017 \mathrm{D}, \mathrm{r}^{2}=0,97$;

c) (toutes les valeurs prises en compte, en dehors de celles correspondant à des niveaux sableux);

$$
\mathrm{Cu}=0,3+0,024 \mathrm{D}, \mathrm{r}^{2}=0,70 \text {. }
$$

En tenant compte de la relation, $\sigma^{\prime}=0,075 \mathrm{D}, \sigma^{\prime}$ contrainte verticale intergranulaire, on en déduit:

a) $\mathrm{Cu}=-0,007+0,236 \sigma^{\prime}$ soit $\frac{\Delta \mathrm{Cu}}{\Delta \sigma^{\prime}}=0,236$.

b) $\mathrm{Cu}=0,03+0,326 \sigma^{\prime}$ soit $\frac{\Delta \mathrm{Cu}}{\Delta \sigma^{\prime}}=0,326$

c) $\mathrm{Cu}=0,14+0,316 \sigma^{\prime}$ soit $\frac{\Delta \mathrm{Cu}}{\Delta \sigma^{\prime}}=0,316$.

Ces relations sont à comparer avec celle de Skempton :

$$
\frac{\mathrm{Cu}}{\sigma^{\prime}}=0,11+0,0037 \mathrm{I}_{\mathrm{p}}
$$

Avec $I_{p}=40$ valeur moyenne des mesures représentées figure 5 , on obtient

$$
\frac{\Delta \mathrm{Cu}}{\Delta \sigma^{\prime}}=0,258
$$

à comparer avec les valeurs obtenues ci-dessus.

A titre d'information, on peut rappeler que pour les vases en fondation du barrage d'Arzal [7] on avait la relation

$$
\frac{\Delta \mathrm{Cu}}{\Delta \sigma^{\prime}}=0,47 \text { (scissomètre) et } 0,40 \text { (laboratoire). }
$$

La cohésion non drainée des vases de Tunis est donc faible, d'autant plus que l'analyse faite n'a pas encore tenu compte de la réduction à apporter aux résultats obtenus au scissomètre, correction qui peut atteindre le tiers des valeurs mesurées [8].

Pour les valeurs résiduelles on a la relation toutes valeurs prises en compte en dehors des lentilles sableuses

$$
\mathrm{Cu}=0,40+0,117 \sigma^{\prime}, r^{2}=0,56
$$

L'examen des résultats montre que certaines valeurs résiduelles sont extrêmement faibles. Ces vases et argiles sont sensibles, plus encore semble-t-il dans les premiers $10 \mathrm{~m}$ que de 10 à $20 \mathrm{~m}$ de profondeur. II faut vraisemblablement chercher une explication à ce phénomène dans le milieu salé dans lequel se sont déposées ces vases et dans la structure qui en résulte. Malheureusement on n'a disposé d'aucune information de ce point de vue sur les échantillons prélevés dans la zone des travaux du canal.

Les valeurs mesurées de la cohésion au cours d'études antérieures essentiellement en laboratoire ne sont pas globalement en contradiction avec les résultats d'essais au scissomètre dont on vient de faire état. 


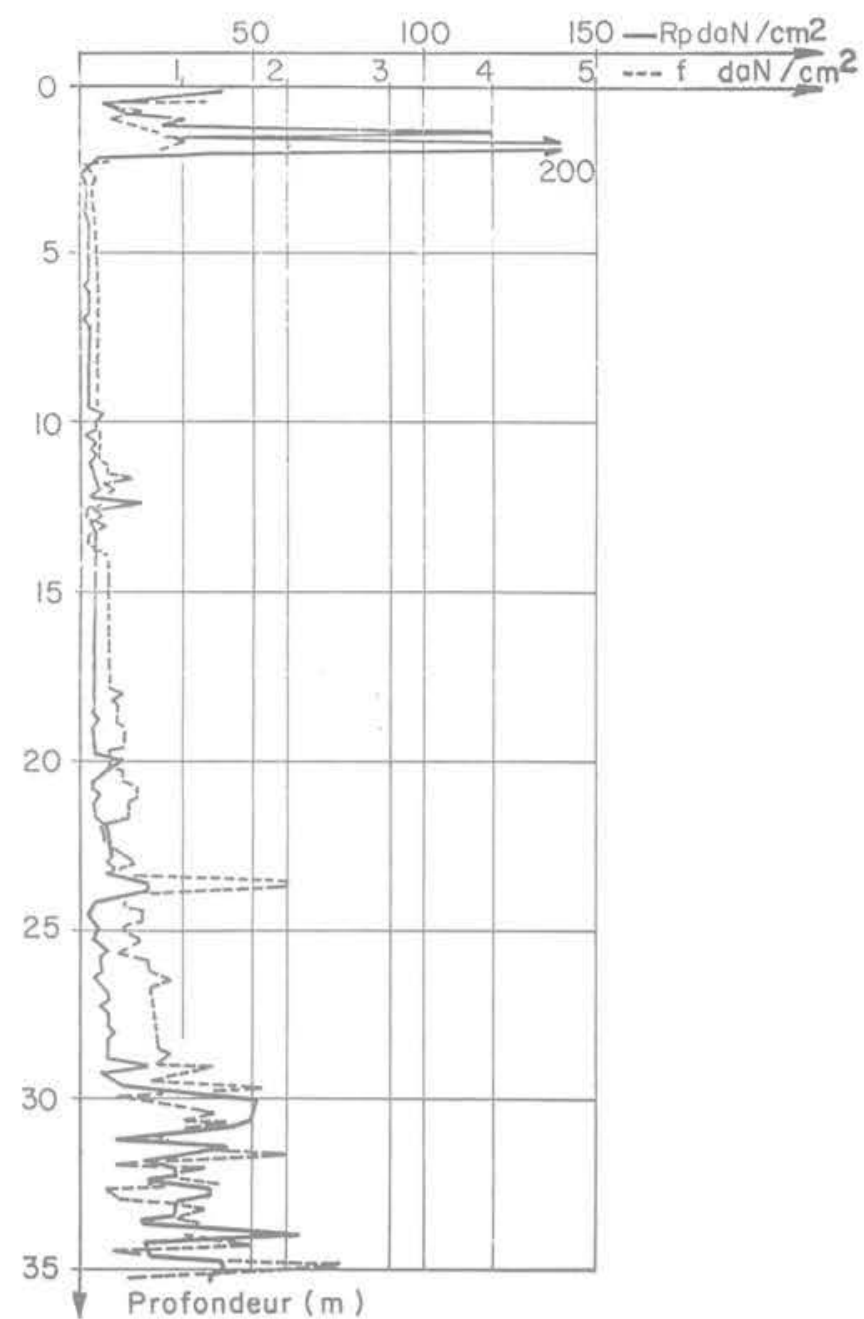

8a Essai pénétrométrique PS2

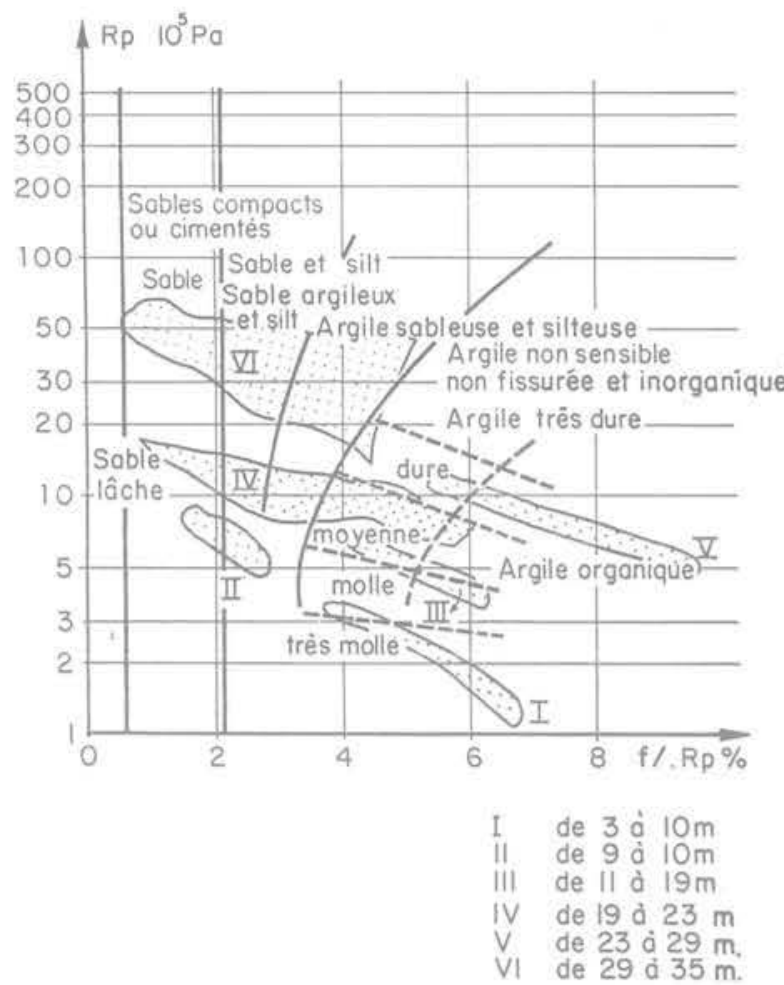

$8 b$ Interprétation dans le diagramme de Schmertmann.

Fig. 8 Essai pénétrométrique PS2

tat. On constate qu'il y aurait eu plusieurs apports de matériaux allant des sables à des matériaux organiques en passant par des argiles. On met en évidence trois séquences, successivement de 35 à $25 \mathrm{~m}$, de 25 à $11 \mathrm{~m}$, de 10 à $3 \mathrm{~m}$ de profondeur.

La figure 9 donne une coupe stratigraphique sur $1300 \mathrm{~m}$ environ de longueur développée. On a mis en évidence les zones sableuses traverseees par les essais pénétrométriques et en complément des zones faiblement sableuses ou surconsolidées détectées par les essais scissométriques.

\section{Compressibilité et consolidation}

En exploitant plusieurs dizaines d'essais de compressibilité à l'œdomètre exécutés sur un ensemble de matériaux argileux prélevés dans les différents horizons que l'on trouve au droit du lac jusqu'à $45 \mathrm{~m}$ de profondeur, on a tenté une corrélation linéaire, avec 43 couples de valeurs, $C_{c}, w_{1}$ (fig. 10), On a obtenu :

$$
C_{c}=0,010\left(w_{L}-21\right) \quad r^{2}=0,69 \text {. }
$$

Ce résultat n'est donné qu'à titre indicatif. II pèche par la qualité de la population des données d'origines très diverses à partir desquelles il a été établi.

Le coefficient de consolidation est connu à partir des informations suivantes: 
a) Un premier groupe d'essais comprenant onze valeurs. II est relatif aux argiles et vases de la zone de Montplaisir, on a les caractéristiques suivantes (Mécasọl 1974) [5] :

Tableau 2

\begin{tabular}{c|c|c|c|c}
\hline & Unité & $\begin{array}{c}\text { Valeurs } \\
\text { moyennes }\end{array}$ & Ecart-type & $\begin{array}{c}\text { Valeurs } \\
\text { extrêmes }\end{array}$ \\
\hline$\gamma \mathrm{d}$ & $\mathrm{kN} / \mathrm{m}^{3}$ & 14,6 & 1,5 & $10,7 / 16,6$ \\
$\mathrm{Cc}$ & & 0,21 & 0,07 & $0,12 / 0,32$ \\
\hline $1+e_{0}$ & $\%$ & 43 & 14 & $32 / 78$ \\
$\mathrm{w}_{\mathrm{L}}$ & $\%$ & 2,37 & 1,42 & $0,8 / 5,4$ \\
$\mathrm{CV}$ & $10^{-3} \mathrm{~cm}^{2} / \mathrm{s}$ & & & \\
\hline
\end{tabular}

La valeur du coefficient de perméabilité est de l'ordre de $5 \cdot 10^{-10} \mathrm{~m} / \mathrm{s}$.

b) Un second groupe comprend quatre valeurs, relatives aux vases dans la zone de l'Esplanade.

On a les résultats suivants (Her 1974) [4] :

Tableau 3

\begin{tabular}{c|c|c|c|c|c|c|c}
\hline $\begin{array}{c}\text { Profondeur } \\
\mathrm{m}\end{array}$ & $\begin{array}{c}\gamma \mathrm{d} \\
\mathrm{kN} / \mathrm{m}^{3}\end{array}$ & $\mathrm{e}_{\mathrm{o}}$ & $\begin{array}{c}\mathrm{w}_{\mathrm{L}} \\
\%\end{array}$ & $\mathrm{Cc}$ & $\frac{\mathrm{Cc}}{1+\mathrm{e}_{\mathrm{o}}}$ & $\begin{array}{c}\mathrm{Cv} \\
10^{-4} \mathrm{~cm}^{2} / \mathrm{s}\end{array}$ & $\begin{array}{c}\mathrm{K} \\
10^{-10} \mathrm{~m} / \mathrm{s}\end{array}$ \\
\hline 9,50 à 10 & 12,3 & 1,20 & 68 & 0,240 & 0,11 & 1,1 & 4 \\
19,75 à 20 & 12,7 & 1,13 & 66 & 0,280 & 0,13 & 1 & 1 \\
7 à 7,50 & 16,1 & 0,68 & 40 & 0,100 & 0,06 & 2 & 1 \\
9,8 à 10,30 & 12,3 & 1,20 & 44 & 0,225 & 0,10 & 1,3 & 1 \\
\hline
\end{tabular}

On notera I'homogénéité des valeurs concernant $C_{v}$. Le domaine de contraintes pour lequel $\mathrm{C}_{\mathrm{v}}$ a été mesuré, est compris entre 0,5 et $1 \cdot 10^{5} \mathrm{~Pa}$.

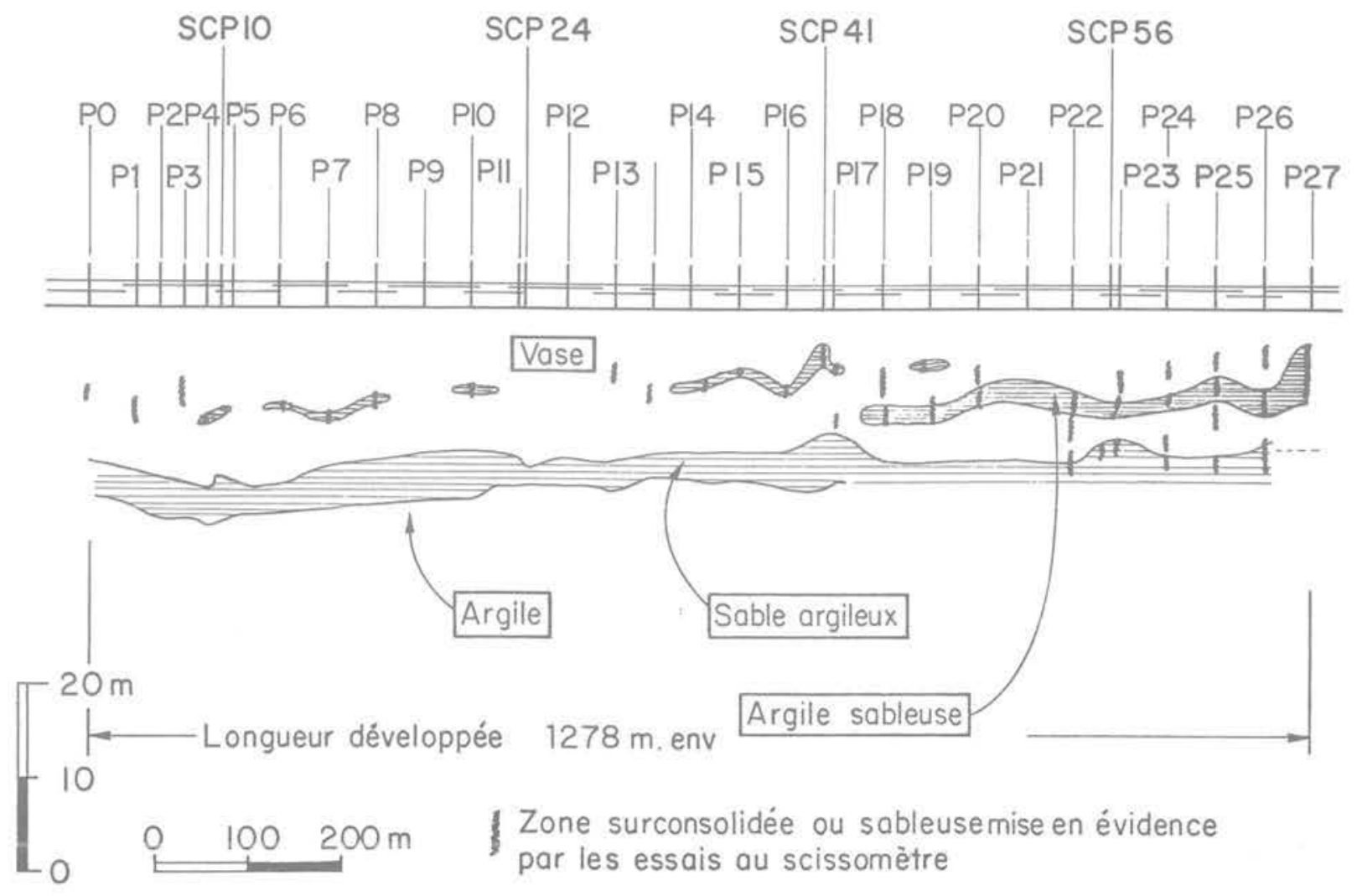

Fig. 9 Coupe stratigraphique de la zone de l'esplanade 
c) Un troisième groupe comprend les valeurs relatives à des vases toujours prélevées dans le lac dans la zone de l'Esplanade.

Tableau 4

\begin{tabular}{c|c|c|c|c|c|c}
\hline $\begin{array}{c}\text { Profondeur } \\
\mathrm{m}\end{array}$ & $\begin{array}{c}\gamma \mathrm{d} \\
\mathrm{kN} / \mathrm{m}^{3}\end{array}$ & $\begin{array}{c}\mathrm{w}_{\mathrm{L}} \\
\%\end{array}$ & $\mathrm{Cc}$ & $\frac{\mathrm{Cc}}{1+\mathrm{e}_{\mathrm{o}}}$ & $\begin{array}{c}\mathrm{Cv} \\
\mathrm{cm}^{2} / \mathrm{s}\end{array}$ & $\begin{array}{c}\sigma \\
10^{5} \mathrm{~Pa}\end{array}$ \\
\hline 7,2 à 7,5 & 11,4 & 78 & 0,68 & 0,25 & $2 \cdot 10^{-4}$ & 0,56 \\
16,2 à 16,8 & 12,2 & 63 & 0,39 & 0,17 & $2 \cdot 10^{-4}$ & 0,56 \\
11,1 à 11,8 & 12,3 & 53 & 0,41 & 0,18 & $2 \cdot 10^{-4}$ & 0,56 \\
\hline
\end{tabular}

Mais, compte tenu des problèmes à résoudre, des mesures de coefficients de consolidation radiale ont été exécutées sur les vases et argiles prélevées dans la zone de l'Esplanade, les résultats obtenus sont les suivants :

Tableau 5

\begin{tabular}{c|r|c|c|c|c|c}
\hline $\begin{array}{c}\text { Profondeur } \\
\mathrm{m}\end{array}$ & $\begin{array}{c}\gamma \mathrm{d} \\
\mathrm{kN} / \mathrm{m}^{3}\end{array}$ & $\begin{array}{c}\mathrm{w}_{\mathrm{L}} \\
\%\end{array}$ & $\mathrm{Cc}$ & $\frac{\mathrm{Cc}}{1+\mathrm{e}_{\mathrm{o}}}$ & $\begin{array}{c}\mathrm{C}_{\mathrm{r}} \\
\mathrm{cm}^{2} / \mathrm{s}\end{array}$ & $\begin{array}{c}\sigma \\
10^{5} \mathrm{~Pa}\end{array}$ \\
\hline 1,2 à 1,80 & 9,0 & 78 & 0,69 & 0,22 & $4 \cdot 10^{-3}$ & 0,65 \\
4,2 à 4,80 & 9,7 & 65 & 0,75 & 0,26 & $8 \cdot 10^{-4}$ & 0,65 \\
4,6 à 5,2 & 8,9 & 81 & 0,60 & 0,20 & $5 \cdot 10^{-3}$ & 0,65 \\
7,9 à 8,5 & 10,4 & 65 & 0,59 & 0,22 & $4 \cdot 10^{-4}$ & 1,3 \\
- & - & - & - & - & $3 \cdot 10^{-4}$ & 0,91 \\
9,6 à 10,1 & 12,0 & 84 & 0,55 & 0,22 & $4 \cdot 10^{-3}$ & 0,65 \\
2 à 2,7 & 9,1 & 77 & 0,75 & 0,23 & $9 \cdot 10^{-3}$ & 0,65 \\
1,2 à 1,8 & 8,9 & 77 & 0,69 & 0,22 & $4 \cdot 10^{-3}$ & 0,65 \\
3,7 à 4,3 & 10,3 & 55 & 0,47 & 0,18 & $4 \cdot 10^{-3}$ & 0,65 \\
1,2 à 1,8 & 9,0 & 80 & 0,78 & 0,25 & $4 \cdot 10^{-3}$ & 0,65 \\
4,2 à 4,7 & 8,1 & 62 & 0,57 & 0,21 & $4 \cdot 10^{-3}$ & 0,65 \\
\hline
\end{tabular}

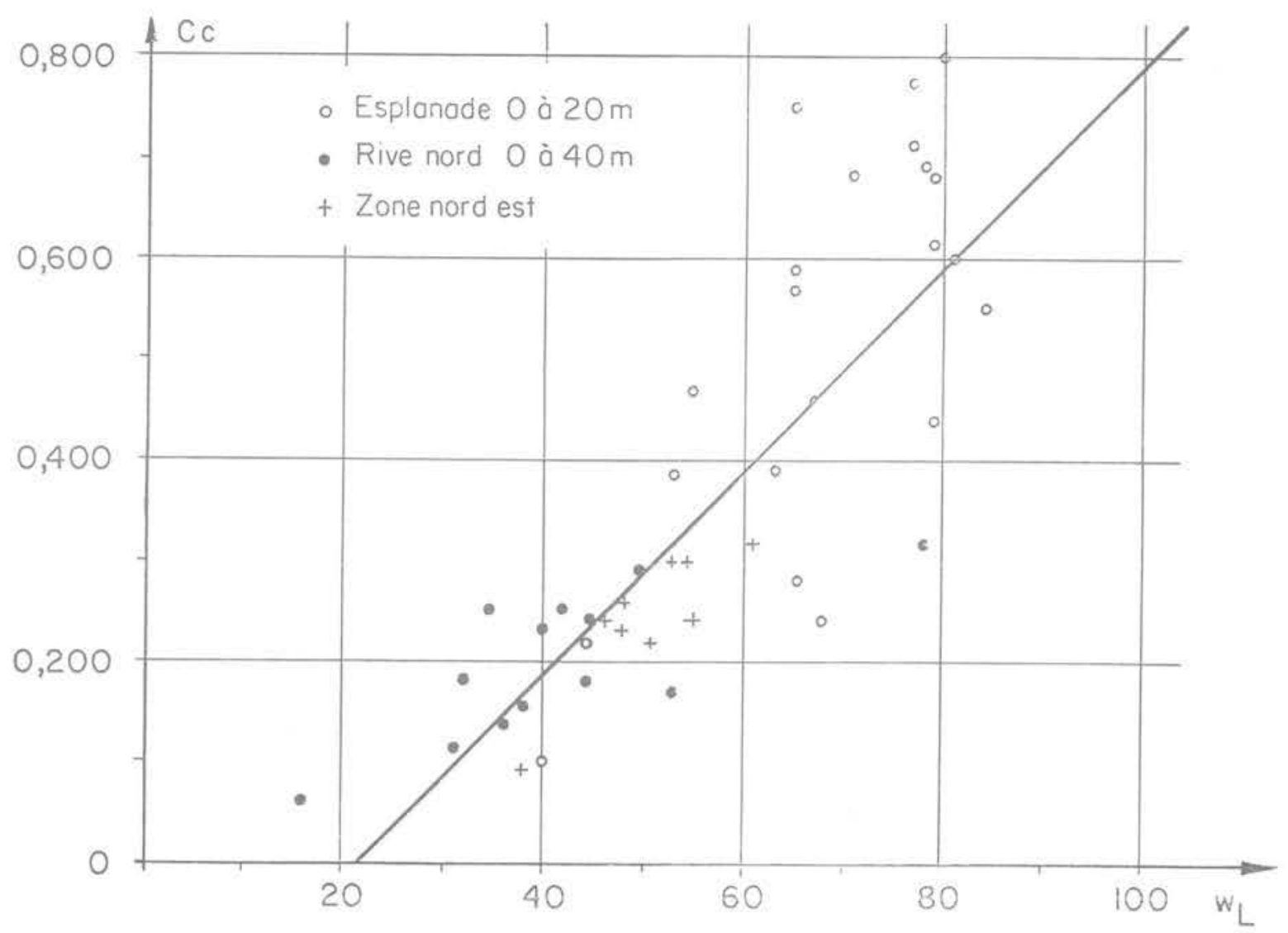

Fig. 10 Coefficient de compressibilité en fonction de la limite de liquidité 
Pour les deux derniers groupes de valeurs relatives à des vases entre 0 et $20 \mathrm{~m}$ de profondeur, pour 13 mesures de $C_{c} /\left(1+e_{o}\right)$, la valeur moyenne est de 0,22 et l'écart-type égal à 0,03 .

Le coefficient de consolidation vertical est de l'ordre de $2 \cdot 10^{-4} \mathrm{~cm}^{2} / \mathrm{s}$ pour un domaine de contrainte de l'ordre de la contrainte verticale qui s'exerce sur les vases en place. On peut vérifier que cette valeur est en accord avec les valeurs données par la relation $\mathrm{C}_{\mathrm{v}}=K E^{\prime} / \gamma \mathrm{w}$.

La valeur moyenne du coefficient de consolidation radiale $C_{r}$ est égale à $3 \cdot 10^{-3} \mathrm{~cm}^{2} / \mathrm{s}$, l'écart-type est $2 \cdot 10^{-3} \mathrm{~cm}^{2} / \mathrm{s}$. Le rapport $\mathrm{C}_{\mathrm{r}} / \mathrm{C}_{\mathrm{v}}$ est voisin de 10 .

\subsubsection{Sables}

Les couches de sable sont constituées de sable fin plus ou moins argileux ou cimenté par des constituants calcaires. Dans le gisement très épais exploité pour fournir le remblai du canal, on a fait de très nombreuses granulomètries. On doit noter la très grande finesse du sable. Le dépôt est très homogène sur 11 à 12 m d'épaisseur, à part quelques passées argileuses peu épaisses. Le $D_{60}$ est au plus égal à $0,20 \mathrm{~mm}$ et le $D_{10}$ toujours de l'ordre de $0,10 \mathrm{~mm}$. La granulométrie est donc très étroite. Dans le paragraphe 1.3.1.3, on a donné une indication sur le domaine de variation du poids volumique sec mesuré sur échantillons. Dans le sondage $\mathrm{S1/74}$ à Tunis Marine, les échantillons sableux ont des densités comprises entre 14,5 et $18,4 \mathrm{kN} / \mathrm{m}^{3}$. Cinq essais en zone d'emprunt ont donné une valeur moyenne de $14,9 \mathrm{kN} / \mathrm{m}^{3}$ avec comme valeurs extrêmes 13,4 et 16,1 . Des essais antérieurs au SPT donnent un angle de frottement de l'ordre de $35^{\circ}$, valeur recoupée par un essai de cisaillement direct sur un échantillon dans le sondage S1/74 à $29 \mathrm{~m}$ de profondeur.

L'examen des résultats des essais au pénétromètre n'est pas en contradiction avec ces valeurs.

\section{Conception générale de l'ouvrage}

\subsection{Contraintes générales - Rappel des condi- tions du site}

L'aménagement général de la zone du rivage du lac parallèle à l'avenue Mohamed- $V$, a exigé que le canal soit réalisé en dehors du remblai existant qui consti tue ce que I'on a coutume d'appeler à Tunis, I'Esplanade. Au contraire de la rive Nord qui est constituée de matériau en place ou rapporté sur une faible épaisseur. matériau médiocre mais mécaniquement suffisant pour l'ouvrage très modeste qu'est le canal; le long de l'Esplanade, on trouve un remblai qui flotte en quelque sorte sur 15 à $20 \mathrm{~m}$ de vase et d'argile molle. Ce remblai est récent. Une grande partie de la basse ville de Tunis a été gagnẻe sur le lac en poussant les déblais les plus variés dans les vases du lac. Les 50 derniers mètres de la bande de terrain entre la nouvelle voie Nord-Sud et le rivage, date de 1925 semble-t-il. L'épaisseur de remblai est de l'ordre de $4 \mathrm{~m}$. Le terrain naturel est à la cote $+1,50$ NGT environ, mais souvent moins et s'abaisse jusqu'à +0.70 alors que le "fond" du lac serait à $-0,50$ environ. La pénétration du remblai serait donc de 2 à 2,50 $\mathrm{m}$ dans les premières couches de vases et de boues du lac. On peut s'aider pour étayer ces affirmations des nombreux sondages et essais au pénétromètre exécutés tout le long du rivage intéressé par le tracé du canal.
En fait, les coupes de certains sondages, en particulier celles des sondages anciens, ne sont pas tout à fait en concordance avec ces affirmations. Cela vient du fait que la notion d'épaisseur et de nature du remblai doit être précisée. On rencontre, en général, d'abord une épaisseur de remblai sans pénétration de vase qui est de l'ordre de $2 \mathrm{~m}$. On trouve ensuite une zone de $2 \mathrm{~m}$ environ de remblai vaseux, c'est-à-dire de mélange de remblai et de vase ou de boue. Cette couche est appelée tantôt remblai, tantôt vase par les sondeurs.

Cette incertitude s'explique d'une part par le mode de mise en place des remblais et d'autre part par la résistance des premières couches d'argile ou de vase qui n'est que de l'ordre de quelques centièmes de $\mathrm{daN} / \mathrm{cm}^{2}$.

Un remblaiement provisoire tout récent dans le lac a provoqué un poinçonnement de $3,50 \mathrm{~m}$ de profondeur environ dans la vase, soit de $3 \mathrm{~m}$ par rapport au niveau du lac. La vase a reflué jusqu'au niveau du remblai qui s'est stabilisé quelques décimètres audessus du niveau du lac. Ceci correspond à une résistance de la vase de l'ordre de $0,05 \mathrm{daN} / \mathrm{cm}^{2}$.

L'expérience montre que l'on peut excaver des ouvrages modestes comme le canal dans le remblai de l'Esplanade maigré la présence de poches de vase due au mode de réalisation du remblai. 50 ans ou plus de consolidation du remblai et de sa fondation expliquent les 0,100 à $0,150 \mathrm{daN} / \mathrm{cm}^{2}$ de résistance au cisaillement non drainé des vases immédiatement sous le remblai.

Ces quelques remarques ont pour but de souligner la difficulté d'exécution d'un remblai en bordure du lac. remblai qui soit homogène et stable.

La recherche de la stabilité à la rupture du remblai pour éviter les conséquences qui en résulteraient sur la qualité du remblai n'est pas le seul problème à résoudre. II ne faut pas que les tassements soient trop importants. II suffit de rappeler que la route Tunis-La Goulette élargie en 1957 a été laissée pendant 10 ans avant que l'on mette la couche de base et le corps de chaussée. Or, 15 ans après environ, les tassements consécutifs de la mise en place de ces deux dernières couches qui représentent une épaisseur de matériau très modeste, sont de l'ordre de plusieurs dizaines de centimètres. Ces tassements sont mis en évidence par les repères que constituent les points durs des appuis du pont de Tunis Marine et des écluses de communication entre le lac Nord et le canal de navigation. Un dernier repère est la conduite Bonna fondée sur pieux au droit de la centrale de La Goulette.

\subsection{Conception générale du remblai}

Le premier problème concernait la nature du remblai. Une recherche de carrière dans la zone de Tunis a conduit à considérer que le matériau le plus adapté et le plus économique serait celui constituant les lentilles de sable que l'on savait géologiquement exister dans le lac le long de la rive Nord. Comme le remblaiement hydraulique était le mode de chargement le mieux adapté à la qualité de la fondation. ces lentilles se prêtaient à une exploitation par dragage et refoulement qui évitait une rupture de charge.

Le second problème concernait la stabilité et le tassement des fondations. La nécessité de réaliser en quelques 18 mois un ouvrage, dont les tassements ultérieurs soient négligeables, a amené à retenir un 


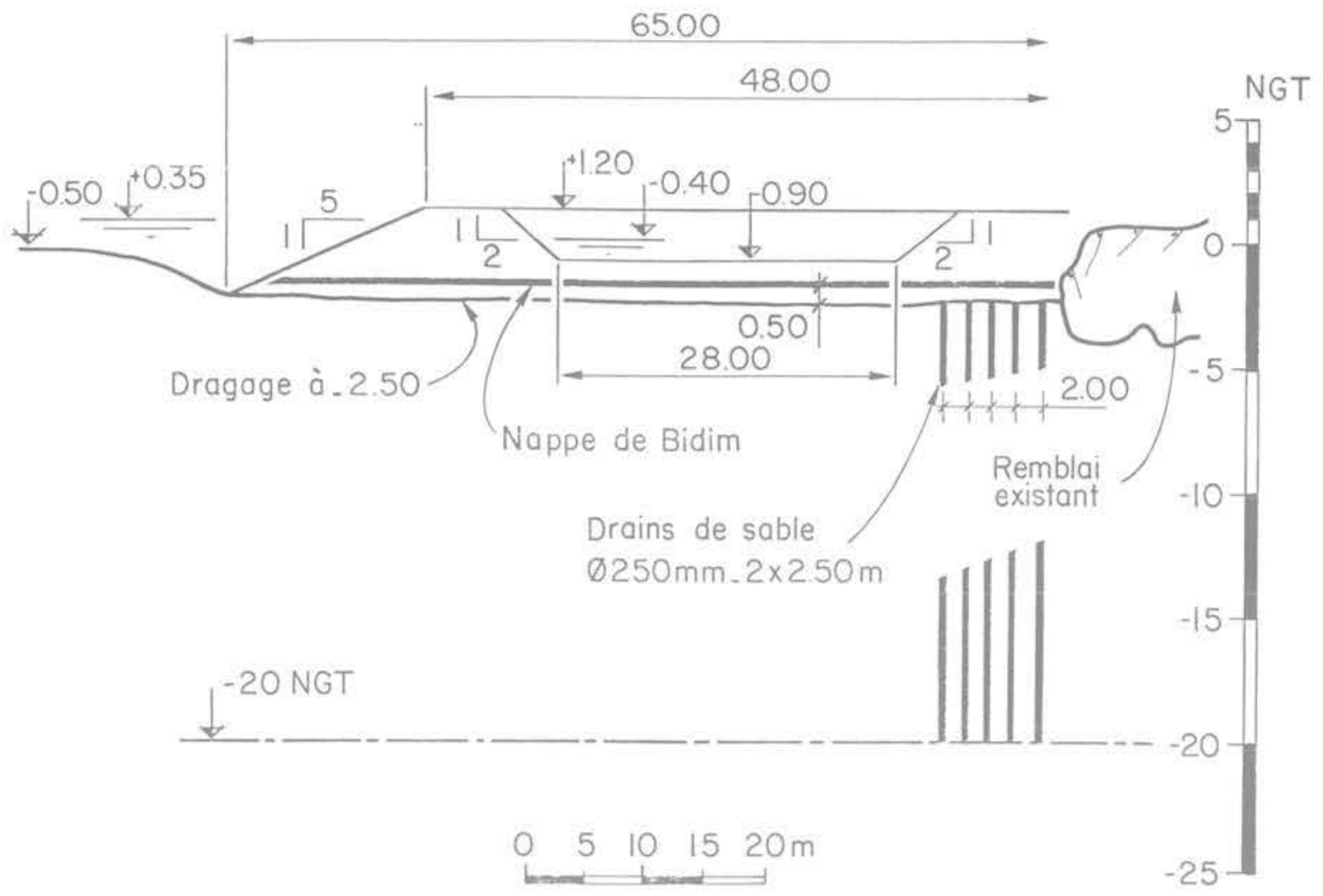

Fig. 11 Coupe type du canal

procédé d'accélération de la consolidation de la fondation du remblai, par drains de sable ou drains de carton. La maitrise de la consolidation était également nécessaire pour améliorer la résistance de la fondation au cours de la construction, afin de ne pas affecter le remblai par une rupture de la fondation, rupture dont ou pouvait difficilement prevoir l'extension et les conséquences.

La satisfaction de ces deux conditions a conduit à écarter toutes les solutions dans lesquelles on surchargeait le sol au-delà de la rupture (explosion, rupture par surcharge, etc.). Toutes les modalités d'exécution étaient commandées par la nécessité de charger progressivement et le plus uniformément possible la fondation.

\subsection{Séquence des opérations de construction}

En fonction du parti adopté, on a prévu de faire les opérations suivantes :

a) Dragage jusqu'à 2,50 NGT de la zone à rembiayer. Cette opération était indispensable pour enlever les vases fermenticides ou très instables qu'il y avait en surface. Aussi paradoxal que cela puisse paraitre, la notion de fond du lac n'était pas une notion claire. Les essais au scissomètre ont permis de déterminer que la cote $-2,50$ NGT était celle à partir de laquelle la résistance au cisaillement non drainée était de l'ordre de quelques centièmes de $\mathrm{daN} / \mathrm{cm}^{2}$ et au moins supérieur à un centième de daN $/ \mathrm{cm}^{2}, 1,50 \mathrm{~m}$ de tirant d'eau était suffisant pour le ponton. Mais on a tenu compte du remblai de $0,50 \mathrm{~m}$, mis préalablement à l'exécution des drains de sable, et de $0.50 \mathrm{~m}$ de profondeur à titre de sécurité. b) Mise en place à l'avancement par remblayage hydraulique de remblai sableux sur une épaisseur de $0.50 \mathrm{~m}$.

On a décidé de la mise en place de ce matelas afin d'assurer la meilleure liaison possible entre les drains et le remblai.

c) Exécution des drains de sable à partir d'un ponton.

d) Mise en place d'une nappe continue transversalement de Bidim U64.

La mise en place de cette nappe a été retenue afin d'améliorer la répartition des charges dues au remblai et de diminuer les contraintes de cisaillement dans la fondation.

e) Remblaiement jusqu'à la cote finale par chargement continu par voie hydraulique sur toute la largeur de la surface à remblayer.

\subsection{Description et justification des dispositions adoptées}

a) Caractéristiques des fondations ayant servi à des calculs de prédimensionnement.

Elles ont été définies à partir d'informations plus réduites que celles dont il est fait état dans le paragraphe 1.

- Volume de matériau à mettre en place : $350000 \mathrm{~m}^{3}$.

- Épaisseur du massif compressible pris en compte dans les calculs : $40 \mathrm{~m}$.

- Accroissement de la résistance au cisaillement : $\Delta \mathrm{C}_{\mathrm{u}}=0,300 \Delta \mathrm{ir}^{\prime}$. 
Rappel maths 2

-

- Caractéristiques à la rupture :

$$
\begin{aligned}
\mathrm{Cu}_{\min } & =0,3 \cdot 10^{4} \mathrm{~Pa} \\
\mathrm{Cu}_{\text {moyen }} & =0,5 \cdot 10^{4} \mathrm{~Pa} \\
\mathrm{Cu}_{\max } & =0,7 \cdot 10^{4} \mathrm{~Pa} .
\end{aligned}
$$

- État de contrainte initial :

Contrainte verticale initiale dans le massif $=0,075 \mathrm{D}$ en $10^{5} \mathrm{~Pa}, \mathrm{D}$ en mètres.

- Caractéristiques de déformations :

de 0 à -19 NGT, $\frac{C c}{1+e_{0}}=0,222$ soit $C c=0,500$,

$\gamma \mathrm{d}=12 \mathrm{kN} / \mathrm{m}^{3}$.

de -19 NGT à -29 NGT, $\frac{\mathrm{Cc}}{1+\mathrm{e}_{0}}=0,177$

soit $\mathrm{Cc}=0,400, \gamma \mathrm{d}=12 \mathrm{kN} / \mathrm{m}^{3}$;

de -19 NGT à -44 NGT, $\frac{\mathrm{Cc}}{1+\mathrm{e}_{\mathrm{o}}}=0,103$

soit $\mathrm{Cc}=0,200, \gamma \mathrm{d}=14 \mathrm{kN} / \mathrm{m}^{3}$.

- Caractéristiques de consolidation :

$$
\mathrm{Cv}=10^{-4} \mathrm{~cm}^{2} / \mathrm{s}, \mathrm{Cr}=5 \cdot 10^{-4} \mathrm{~cm}^{2} / \mathrm{s} \text {. }
$$

On a admis que les argiles étaient normalement consolidées.

b) Caractéristiques de l'ouvrage (figure 11):

- cote de départ de la fondation : $-2,50$ NGT:

- cote finale du remblai : +1,20 NGT:

- largeur en crête finale : $48,00 \mathrm{~m}$ :

- talus côté lac : $5 / 1$;

- maille des drains de sable : $2 \times 2,50 \mathrm{~m}$;

- diamètre des drains : $250 \mathrm{~mm}$;

- cote de l'extrémité des drains : -20 NGT:

- longueur totale des drains : $335000 \mathrm{ml}$. c) Modalités d'exécution

Pour une charge finale du remblai égale à $0,57 \cdot 10^{5} \mathrm{~Pa}$, le tassement total prévu dans l'axe du remblai a été estimé à environ $1,50 \mathrm{~m}$. La charge initiale admissible sur la fondation a été fixée à $1,70 \mathrm{~m}$ de remblai immergé. Compte tenu des caractéristiques de consolidation, la montée ultérieure du remblai a été limitée à $0,50 \mathrm{~m}$ toutes les six semaines. $\mathrm{La}$ durée totale de remblaiement en un point est donc de 12 mois et la durée du chargement après remblaiement et avant excavation du canal, est de 4 mois.

II n'a pas été possible pour différentes raisons ni d'avoir toutes les informations souhaitables sur les sols, ni surtout, de procéder à l'exécution d'un remblai d'essai. Aussi s'est-on délibérément placé du côté de la sécurité. On a prévu une auscultation de la partie du remblai à exécuter en début des travaux, c'est-à-dire côté Tunis Marine. Le but recherché était d'en faire non une section d'essai, mais une section pilote qui permette de conduire au mieux les travaux et en particulier de régler la vitesse de mise en place du remblai qui était le seul paramètre qu'il était possible de modifier.

\section{Conditions d'exécution}

\subsection{Drains de sable (fig. 12)}

On avait laissé le choix possible entre des drains de sable et des drains carton ou équivalent. La comparai son économique a été défavorable au drain du second type. Ceci est dû aux conditions économiques locales.

Les drains de sable ont été exécutés au moyen d'un ponton de $71 \mathrm{~m}$ de longueur et de $8,90 \mathrm{~m}$ de largeur. Sur un côté, le ponton est équipé de 34 tubes guides de $5 \mathrm{~m}$, distant entre eux de $2 \mathrm{~m}$. Ces tubes avaient pour but principal la traversée des vases en tête des

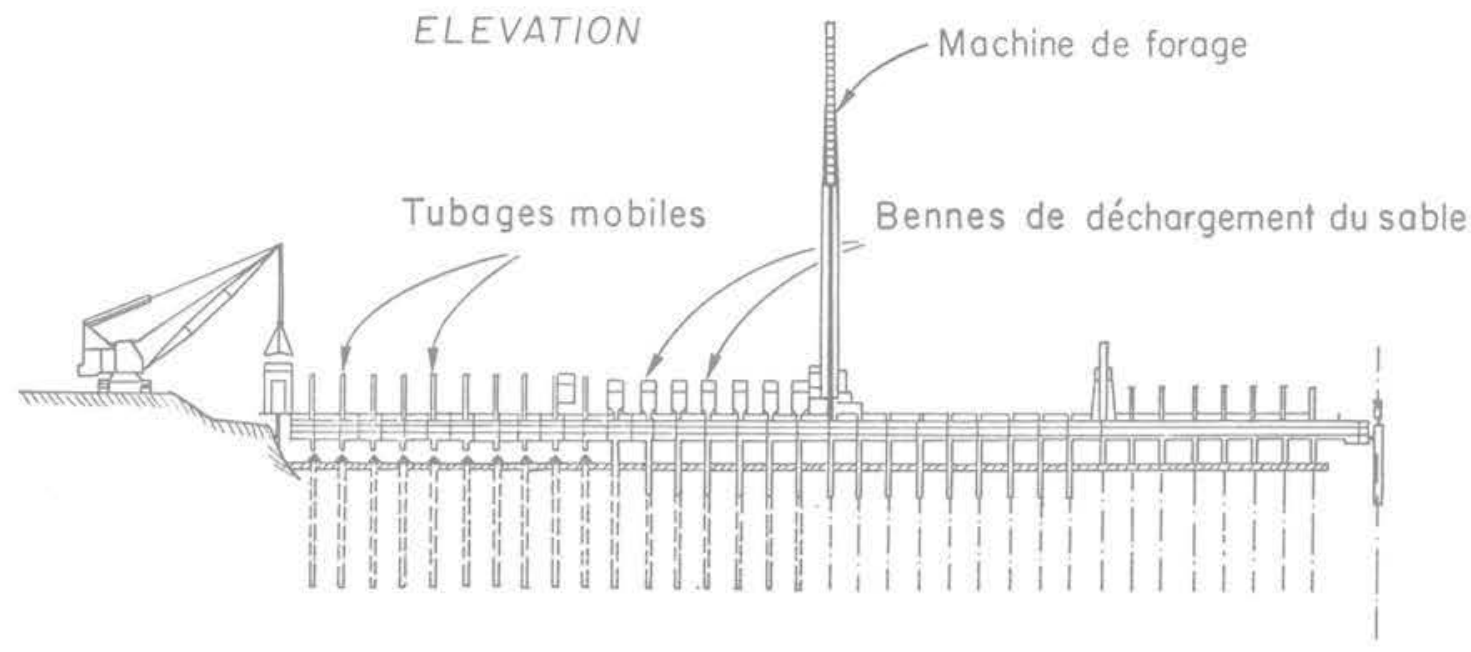

VUE EN PLAN

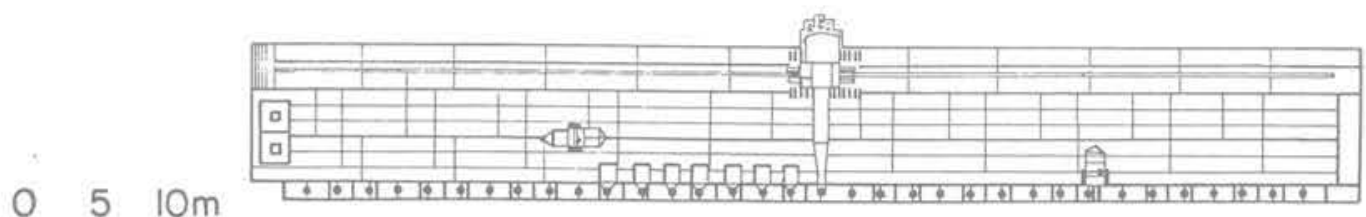

Fig. 12 Ponton ayant servi à l'exécution des drains de sable 
dépôts et le remplissage du forage. Ils sont répartis sur une longueur de $68 \mathrm{~m}$, permettant de réaliser ainsi une ligne de drains sur la largeur du remblai. Audessus de chaque tube peuvent se mettre en place des bennes pour l'alimentation en sable. Sur le pon ton circule une grue équipée d'une glissière verticale sur laquelle coulisse l'engin de perforation.

La succession d'opération est la suivante. Le tube est descendu et pénètre de $2,50 \mathrm{~m}$ dans la vase. La perforation du drain est réalisée par un outil du type "queue de carpen. L'évacuation des sédiments est faite au moyen d'injection d'eau sous pression au cours de la perforation.

Les sédiments en émulsion sont alors recueillis dans une goulotte placée le long du ponton. Repris par une pompe à l'extrémité du ponton, les sédiments sont envoyés en dehors de la zone d'emprise des ouvrages.

Le remplissage est fait par mise en place du sable par gravité. Au cours de sa descente, le sable se bloque par effet de voûte que l'on détruit par injection d'eau. On vérifie le volume de sable mis en place. On remarque un quart d'heure après le remplissage, une descente du sable de l'ordre de $50 \mathrm{~cm}$ correspondant à la mise en place définitive du drain. En cours de remplissage, une mèche de Bidim est placée à la cote - 2,25. Elle aura pour but d'assurer, si nécessaire, la continuité du drainage entre le drain et la couche de sable de $0,50 \mathrm{~m}$ déjà mise en place.

Le forage du trou demande $5 \mathrm{~min}$, le remplissage $14 \mathrm{~min}$. Le programme prévoyait l'exécution de 90 pieux daris la journée (poste de 10 heures). De 70 pieux par jour pendant la période de mise au point, les rendements ont progressivement augmenté jusqu'à atteindre 120 pieux par jour.

Le sable employé était du sable de carrière 0 à $2 \mathrm{~mm}$ $\left(D_{10}=0,2 \mathrm{~mm}, D_{60}=0,5\right.$ à $\left.1 \mathrm{~mm}\right)$.

\subsection{Exploitation de la zone d'emprunt et mise en place du remblai}

La zone d'emprunt est exploitée au moyen d'une drague suceuse capable de draguer des couches jusqu'à $15 \mathrm{~m}$ de profondeur. Le gros problème a été le déroctage de la croûte calcaire de surface qui a exigé l'emploi d'une pelle Poclain 300 , après un échec avec une Poclain 150 installée sur un ponton ancré par deux pieux. Elle assurait un rendement journalier de $500 \mathrm{~m}^{2}$.

La drague est équipée d'un moteur de $1200 \mathrm{CV}$. Elle peut débiter $150 \mathrm{~m}^{3}$ /heure et le débit solide correspond à une concentration de l'ordre de $15 \%$. Le sable est transporté sur le site du remblai par une conduite de refoulement de $270 \mathrm{~mm}$ de diamètre et d'une longueur de $6 \mathrm{~km}$. II y a deux stations de reprise de $700 \mathrm{CV}$. Un tiers de la longueur de la conduite est posée sur flotteur sur le lac. Le reste est à terre. II n'a pas été possible de diminuer la longueur totale, car la profondeur du lac trop faible en de nombreux endroits n'a pas permis la mise en place de toute la conduite sur flotteur.

On doit remarquer que le sable en place est compact. Les talus en zone d'emprunt se tiennent verticaux sur plusieurs mètres. Le sable est mis en place au moyen d'un diffuseur qui se déplace transversalement sur l'emprise du canal poussé par une vedette. La production est de $1000 \mathrm{~m}^{3} /$ jour de remblai en place sur 2 postes et doit atteindre $1200 \mathrm{~m}^{3} /$ jour avant la fin du chantier. Le poids volumique sec du remblai n'a été mesuré que par quelques essais. II est compris entre 16.7 et $17.3 \mathrm{kN} / \mathrm{m}^{3}$. Ces valeurs seraient à exprimer en densité relative. Elles correspondent a priori à des valeurs obtenues dans des conditions analogues [10]. Ce qui correspond à une densité immergée comprise entre 1,05 et 1,09 . Les talus côté lac se sont établis à $5 / 1$.

\subsection{Mise en place du Bidim}

Le Bidim en rouleau est posé sur un ponton. II a été préalablement mouillé. II est mis en place par déroulement. Le ponton se déplace au moyen de 4 treuils, deux raccordés à des points à terre, deux à des corps morts au large. Le repérage se fait par alignement sur la rive. Le recouvrement de deux lais est d'un mètre. On a préféré ceci à une couture. Une des difficultés de mise en place du Bidim vient du fait qu'il flotte. II faut le lester avec 8 à 10 sacs de sable tous les mètres. Le Bidim est imperméable au gaz, ce qui ne facilite pas sa mise en place. Pour éviter les difficultés, il faut faire dans la nappe de géotextile des clapets au moyen de découpure triangulaire, à raison d'une tous les $5 \mathrm{~m}$. On repère les languettes par les bulles de gaz ou d'air qui remontent à la surface de l'eau.

L'avancement est de $25 \mathrm{~m}$ par jour. Le Bidim est ancré sur la rive.

\section{Observations du comportement}

\subsection{Système d'auscultation (fig. 13)}

Il comprend sur $100 \mathrm{~m}$ de longueur dans la zone dite "d'essai " une série de profils comportant chacun :

- 2 cellules de tassement LCPC cousues sur la nappe de Bidim à la base du remblai, dite cellules de surface. Ces cellules sont situées de part et d'autre de l'axe du remblai :
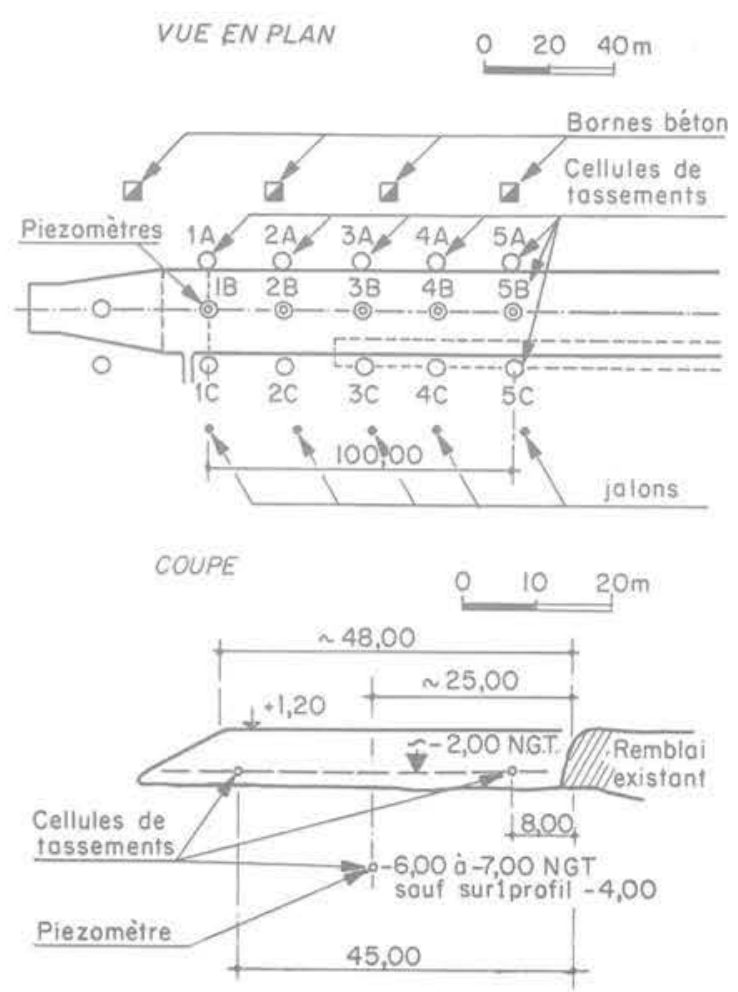

Fig. 13 Auscultation de la tête du remblai 
- 1 cellule de tassement dite de "profondeur " à 6 à $7 \mathrm{~m}$ de profondeur dans l'axe du remblai, sauf pour un profil où elle se trouve à $3 \mathrm{~m}$ de profondeur:

- 1 cellule de mesure de pression interstitielle LCPC piacée au même niveau que la cellule de tassement de profondeur:

- 1 borne en béton sur la rive:

- 1 jalon au pied du talus côté lac.

Au-delà de cette zone ainsi équipée on a disposé tous les $50 \mathrm{~m}$ :

- une ou deux cellules de surface;

- un jalon en pied de talus côté lac:

tous les $120 \mathrm{~m}$ :

- un plot en béton sur la rive:

tous les $300 \mathrm{~m}$ environ

- une cellule de "profondeurn;

- une cellule de mesure de pression interstitielle.

\subsection{Observations}

On a suivi le déplacement des cellules et la variation de la pression interstitielle. Des nombreux graphiques qui ont été tracés on a retenu celui de la figure 14 comme exemple du comportement de la fondation du remblai dans la zone d'essais.

Les tassements, alors que la charge est de l'ordre de $0,38 \cdot 10^{5} \mathrm{~Pa}$ soit $75 \%$ de la charge finale sont compris entre 24 et $67.9 \mathrm{~cm}$. La moyenne est de $34,2 \mathrm{~cm}$ avec un écart type de 12 (12 valeurs pour 6 profils).

\subsection{Interprétation des observations}

\subsubsection{Généralités}

II faut rappeler que les observations n'avaient pour but que de diriger la construction du remblai au plan de la vitesse et de la sécurité. On pourra regretter de ne pas avoir pu profiter de ces travaux pour mettre un plus grand nombre d'appareils ou faire des observations plus fréquentes.

\subsubsection{Tassements}

A priori, on peut, en examinant les courbes de tassement en fonction du temps, s'étonner de voir qu'il n'y a pas correspondance entre l'importance des chargements successifs et les tassements globaux de la fondation mesurés par les cellules de surface.

On a les éléments suivants :

On a distingué 3 chargements après la mise en place de la $1^{\text {re }}$ couche à $0,50 \mathrm{~m}$ avant la pose du Bidim.

\begin{tabular}{c|c|c|c|c}
\hline Chargement & Remblai & Valeurs extrêmes & $\begin{array}{c}\text { Tassement }(\mathrm{cm}) \\
\text { moyen }\end{array}$ & Écart-type \\
\hline $1^{\text {er }}$ & $1,20 \mathrm{~m}$ soit $0,13 \cdot 10^{5} \mathrm{~Pa}$ & $6,5 / 33,2$ & 6,8 & 7,7 \\
$2^{\mathrm{e}}$ & $0,30 \mathrm{~m}$ soit $0,03 \cdot 10^{5} \mathrm{~Pa}$ & $4,1 / 15,3$ & 8,1 & 3,9 \\
$3^{\mathrm{e}}$ & $1,50 \mathrm{~m}$ soit $0,19 \cdot 10^{5} \mathrm{~Pa}$ & $1 / 33,3$ & 10 & 8,6 \\
\hline
\end{tabular}

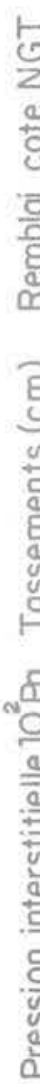

Bidim

100

0.00

$-1.00$

10

20

30

40

50

60

70

용 9,20

9,00

8,80

8,60

8,40

8,20

8,00

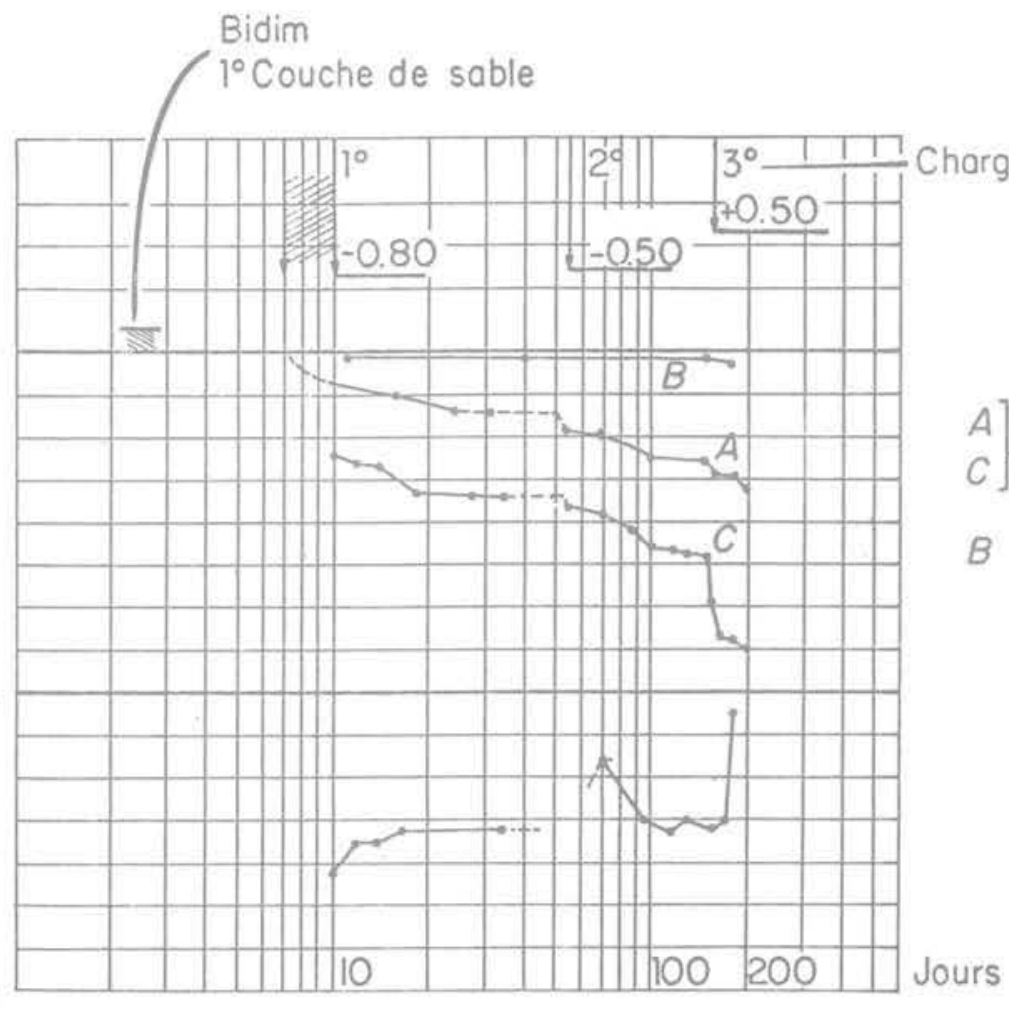

$\left.\begin{array}{l}A \\ C\end{array}\right]$. Cellule de surface

$B$ Cellule de profondeur

Fig. 14 Tassements - Profil 2 -Observations 
Une première explication des écarts constatés tient au fait que le niveau supérieur du remblai n'a pas été aussi uniforme qu'il aurait dû l'être.

La seconde explication résulte du fait que chacune des couches n'a pas eu l'épaisseur moyenne annoncée. En incluant les 50 premiers centimètres de sable et avec $35 \mathrm{~cm}$ de tassement, $75 \%$ environ de la charge finale a été mise en place.

Malgré de grosses différences dans les tassements mesurés, les cellules de surface d'un même profil comme on peut le voir sur la figure 14 ont eu toujours qualitativement le même comportement.

Il est très important de souligner que seuls les 5 à 6 premiers mètres ont été le siège de tassements importants. Les cellules de profondeur n'ont enregis tré que des tassements de 2,3 à $3,7 \mathrm{~cm}$ si l'on excepte celle du profil $4.9 \mathrm{~cm}$. Mais alors que toutes les cellules précédentes sont situées entre 5,30 à $6 \mathrm{~m}$ sous le niveau du Bidim, cette dernière cellule n'est qu'à $2.80 \mathrm{~m}$ de profondeur.

Pour les 3 chargements, le module moyen de la couche supérieure est de $7.8 \cdot 10^{5} \mathrm{~Pa}$ avec un écarttype de $1.8 \cdot 10^{5} \mathrm{~Pa}$. Cet ordre de grandeur est en accord avec les caractéristiques moyennes des matériaux.

On doit noter que les bornes en béton sur le rivage n'ont pratiquement pas bougé : tassement de quelques millimètres seulement.

\subsubsection{Pressions interstitielles}

Les lectures des valeurs des pressions interstitielles sont difficiles à interpréter; les surpressions interstitielles sont faibles : quelques dizaines de millibars. Elles correspondent sensiblement à l'augmentation du tassement (voir fig. 14) et semblent se dissiper rapidement. Ces lectures n'ont été corrigées ni des variations de niveau du lac ni de celles de la pression atmosphérique. Néanmoins, le troisième chargement montre une montée de pressions interstitielles pour 5 profils compris entre 35 et 55 millibars, soit en moyenne 47 millibars.

En admettant que le dernier chargement correspond à une contrainte de l'ordre de $0,19 \cdot 10^{5} \mathrm{~Pa}$, on obtient une valeur de $\bar{B}$ de l'ordre de 0,25 . II ne faut pas oublier que les mesures de pressions interstitielles sont très perturbées par la proximité des drains.

\subsubsection{Consolidation}

II est indéniable que la consolidation de la fondation est rapide et effective. L'observation conjointe des courbes de tassements et de variations de la pression interstitielle permet d'estimer que le temps correspondant à $50 \%$ de consolidation serait compris entre 10 et 20 jours. On obtient une valeur de $C_{r}$ variant de 9 à $4.5 \cdot 10^{-3} \mathrm{~cm}^{2} / \mathrm{s}$, ceci pour un niveau de contrainte faible.

En considérant la valeur moyenne de $6 \cdot 10^{-3} \mathrm{~cm}^{2} / \mathrm{s}$, on constate que le coefficient de consolidation radiale serait plus élevé que celui mesuré en laboratoire, ce qui n'a rien d'étonnant compte tenu du problème du remaniement qui intervient dans le prélèvement des argiles molles.

Les lentilles millimétriques de sable peuvent perturber l'interprétation que l'on peut faire des mesures. Car on sous-estime le diamètre efficace des drains.

\section{Conclusions}

Un dernier chargement reste à faire et il apportera des enseignements utiles qui permettront de mieux con naitre un matériau qui concerne beaucoup de projets à Tunis.

Toutefois, on peut avancer les conclusions suivantes :

II est vraisemblable qu'en fin de chargement du remblai on n'atteindra pas les tassements calculés et la consolidation du matériau est beaucoup plus rapide que celle escomptée. On savait qu'il en serait ainsi mais on ignorait dans quelle proportion. Des tassements inférieurs permettent de diminuer le volume de remblai. L'accélération de la consolidation a servi à augmenter la vitesse de mise en place du remblai et accroît sensiblement la sécurité de la fondation à la rupture.

L'explication de ces écarts tient aux effets du remaniement des échantillons d'argile et de vase molle, sur la mesure des propriétés à la déformation. Ceci concerne la détermination de la pression de préconsolidation et la mesure des caractéristiques de consolidation.

Le niveau de contrainte appliqué jusqu'à maintenant est faible. Une grande partie de la fondation se comporte comme un matériau surconsolidé. Les tas sements sont bien moindres et la consolidation beaucoup plus rapide [11]. C'est ce que montrent les valeurs des tassements observés. Seule la vase des premiers mètres se comporte comme un matériau normalement consolidé, les valeurs plus discutables des mesures de pression interstitielle vont néanmoins dans le même sens.

Ces résultats rendent plus claire la lecture du graphique de la figure $8 \mathrm{~b}$. Ils montrent l'influence de la consolidation intervenue au cours des différentes phases de remplissage du lac. Le comportement de la fondation est en accord aussi avec les résultats des essais aux scissomètres.

Toutefois, on n'a pas déterminé le niveau de préconsolidation des vases et argiles du lac car les sollicitations appliquées jusqu'alors sont faibles et seul un chargement in situ jusqu'au niveau adéquat peut donner des indications valables. Enfin la sensibilité du matériau doit conduire à la prudence. La structure de l'argile a limité ses déformations dans la mesure oủ les sollicitations ont été faibles et les surpressions interstitielles bien drainées. Mais il ne faudrait pas pour autant surestimer les possibilités du matériau.

Nous remercions monsieur Delloua, Président-Directeur général de l'Office National de l'Assainissement à Tunis, pour avoir autorisé la présente publication.

Nous ne saurions terminer ce texte sans remercier toutes les entreprises participant aux travaux du canal Lac Nord et en particulier la Société Internationale de Dragages et de Travaux Hydrauliques, filiale française de P. V. W., Hollande, associée à W. M. N. qui a réalisé les drains de sable, et Cogema Tunis, qui exécute les travaux de remblaiement.

Les reconnaissances in situ et essais de laboratoire particuliers au canal et le suivi du comportement du remblai ont été faits par la Direction des Études et Grands Travaux Hydrauliques du Ministère de I'Agriculture de la République Tunisienne et la Société Fondasol. 


\section{Définition des notations}

$\sigma^{\prime}$

$E^{\prime}$

$\gamma w$

$\gamma d$

e

$w_{L}$

$W_{P}$

$I_{p}$

D

$D_{n}$

k

$\mathrm{C}_{\mathrm{c}}$

$\sigma^{\prime} v_{0}$

$\sigma^{\prime} p$

$\mathrm{C}_{\mathrm{u}}$

$r^{2}$

$\mathrm{c}_{\mathrm{v}}$ contrainte effective normale

module œdométrique

poids volumique de l'eau

poids volumique du sol sec

indice des vides

limite de liquidité

limite de plasticité

indice de plasticité

diamètre de grain

diamètre à $n$ pour cent

coefficient de perméabilité

indice de compression

contrainte effective verticale

initiale en place

pression de préconsolidation

cohésion apparente

coefficient de corrélation

coefficient de consolidation

\section{Références bibliographiques}

[1] PIMIENTA J. * Le cycle Pliocène actuel dans les bassins paraliques de Tunis " - Mémoires de la Société Géologique de France, Tome XXXVIII - Mémoire n 85 Paris - 1959

[2] HABRIDGE W., PILKEY O. H., WHALKING P. SWETLAND P. \& Sedimentation in the lake of Tunis : A lagoon strongly influenced by man " - Environmental Geology - Vol. 1 - Springer-Verlag, New York - 1976.

[3] «Aménagement et Assainissement des lacs Sedjoumi et Tunis" - Rapport, Ingénieurs-Conseils néerlandais, ICN - Mai 1962 - pour le compte du Ministère de l'Agriculture, Direction EGTH - Tunis.

[4] Document d'Archives du Ministères de l'Agriculture, Direction EGTH. Tunis, relatifs aux études d'assainissement du Lac de Tunis 1966-1974.

[5] Documents d'Archives du Ministère de l'Équipement - Tunis - relatifs à la liaison Nord-Sud, route Tunis-La Goulette. Aménagement du quartier de la Petite Sicile 1967-1974.

[6] Documents d'Archives de l'Office National d'Assainissement ONAS - Tunis - 1974-1980.

[7] KERISEL. J. "Le barrage d'Arzal, un barrage sur sol compressible, construit au travers d'un estuaire à marée” - Géotechnique n 23 - 1973 - pages 49-65.

[8] BJERRUM J. (Problems of soils mechanics and construction on soft clays and structurally instable soils" - Session spéciale no 4 - Congrès International de Mécanique des Sols et Fondations - Vol. 3 Moscou, U.R.S.S. - 1973 - pages 111-159.

[9] SANGLERAT G. *The Penetrometer and soil exploration - Developments in geotechnical engineering" Elsevier Publishing Company - Amsterdam 1972.

[10] WHITMAN R. V. "Hydraulic fills to support structural load" - Specialty Conference "Placement and improvement of soil to support structures" - A.S. C.E. - Cambridge - 1968.

[11] LEROUEIL S., TAVENAS F., MIEUSSENS C., PEIGNAUD M. "Construction pore pressures in clay foundations under embankments, Part II : generalized behaviour" - Canadian Geotechnical Journal - Vol. 15 - 1978 